\begin{tabular}{|c|c|}
\hline Title & Structure theorems for vector valued Siegel modular forms of degree 2 and weight det(k) circle times Sym (10) \\
\hline Author(s) & Takemori, Sho \\
\hline Citation & $\begin{array}{l}\text { International journal of mathematics, 27(12), } 1650101 \\
\text { https://doi.org/10.1142/S0129167X 16501019 }\end{array}$ \\
\hline Issue Date & 2016-11 \\
\hline DOC URL & http:/hdl.handle.net/2115/67521 \\
\hline Rights & $\begin{array}{l}\text { Electronic version of an article published as International Journal of Mathematics, } 27,12,2016 \text {, } \\
10.1142 / 20129167 \text { 16501019 @ copyright W orld Scientific Publishing Company } \\
\text { http:/www.worldscientific.com/doi/abs/10.1142/S0129167X 16501019 }\end{array}$ \\
\hline Type & article (author version) \\
\hline File Information & sym10str.pdf \\
\hline
\end{tabular}

Instructions for use 
International Journal of Mathematics

(C) World Scientific Publishing Company

\title{
Structure theorems for vector valued Siegel modular forms of degree 2 and weight $\operatorname{det}^{k} \otimes \operatorname{Sym}(10)$
}

\author{
Sho Takemori \\ Department of Mathematics, Hokkaido University \\ Kita 10, Nishi 8, Kita-Ku, Sapporo, 060-0810, Japan \\ E-mail: takemori@math.sci.hokudai.ac.jp
}

\begin{abstract}
We prove the explicit structure theorems of modules $\bigoplus_{k} M_{\operatorname{det}^{k} \otimes \operatorname{Sym}(10)}\left(\operatorname{Sp}_{2}\left(\mathbb{Z}_{2}\right)\right)$ of vector valued Siegel modular forms of degree 2, where $k$ runs over the set of even integers or odd integers. We also check the conjecture given by Ibukiyama 7 for modules of vector valued Siegel modular forms of degree 2 of weights $\operatorname{det}^{*} \otimes \operatorname{Sym}(8)$ and $\operatorname{det}^{*} \otimes \operatorname{Sym}(10)$.

Keywords: structure theorems of modular forms, vector valued Siegel modular forms, determinant of Siegel modular forms

Mathematics Subject Classification 2010: 11F46, 11F11
\end{abstract}

\section{Introduction}

For $k, j \in \mathbb{Z}_{\geq 0}$, we denote by $M_{k, j}\left(\Gamma^{(2)}\right)$ the space of vector valued Siegel modular forms of weight $\operatorname{det}^{k} \otimes \operatorname{Sym}(j)$. Here $\operatorname{Sym}(j)$ is the symmetric tensor representation of degree $j$ of $\mathrm{GL}_{2}(\mathbb{C})$. Let $A_{\mathrm{ev}}$ be the ring of scalar valued Siegel modular forms of degree2, level 1 and even weights:

$$
A_{\mathrm{ev}}=\bigoplus_{k \in 2 \mathbb{Z}} M_{k}\left(\Gamma^{(2)}\right)
$$

where $M_{k}\left(\Gamma^{(2)}\right)=M_{k, 0}\left(\Gamma^{(2)}\right)$. For $j \in \mathbb{Z}_{\geq 0}$ and $\epsilon=0,1$, we define the graded $A_{\mathrm{ev}}$ module of vector valued Siegel modular forms as

$$
M_{\text {Sym }(j)}^{\epsilon}\left(\Gamma^{(2)}\right)=\bigoplus_{k \equiv \epsilon \bmod 2} M_{k, j}\left(\Gamma^{(2)}\right) .
$$

For a graded module $M=\sum_{k \in \mathbb{Z}} M_{k}$ and an integer $a$ define $M(a)$ by $M(a)_{k}=$ $M_{a+k}$. Roughly speaking, our first main theorem is stated as follows.

Theorem 1.1. As a grated $A_{\mathrm{ev}}$ module, $M_{\mathrm{Sym}(10)}^{0}\left(\Gamma^{(2)}\right)$ has the following free resolution.

$$
\begin{aligned}
& 0 \rightarrow A_{\mathrm{ev}}(-24) \oplus A_{\mathrm{ev}}(-26) \rightarrow \\
& \quad A_{\mathrm{ev}}(-6) \oplus A_{\mathrm{ev}}(-8) \oplus A_{\mathrm{ev}}(-10)^{2} \oplus A_{\mathrm{ev}}(-12)^{2} \oplus A_{\mathrm{ev}}(-14)^{3} \\
& \quad \oplus A_{\mathrm{ev}}(-16)^{2} \oplus A_{\mathrm{ev}}(-18) \oplus A_{\mathrm{ev}}(-20) \rightarrow M_{\mathrm{Sym}(10)}^{0}\left(\Gamma^{(2)}\right) \rightarrow 0 .
\end{aligned}
$$


As a grated $A_{\mathrm{ev}}$ module, $M_{\mathrm{Sym}(10)}^{1}\left(\Gamma^{(2)}\right)$ has the following free resolution.

$$
\begin{aligned}
& 0 \rightarrow A_{\mathrm{ev}}(-27) \oplus A_{\mathrm{ev}}(-29) \rightarrow \\
& \quad \begin{array}{l}
A_{\mathrm{ev}}(-9) \oplus A_{\mathrm{ev}}(-11) \oplus A_{\mathrm{ev}}(-13) \oplus A_{\mathrm{ev}}(-15)^{3} \oplus A_{\mathrm{ev}}(-17)^{3} \\
\oplus A_{\mathrm{ev}}(-19)^{2} \oplus A_{\mathrm{ev}}(-21) \oplus A_{\mathrm{ev}}(-23) \rightarrow M_{\mathrm{Sym}(10)}^{1}\left(\Gamma^{(2)}\right) \rightarrow 0 .
\end{array}
\end{aligned}
$$

Moreover maps in the free resolutions can be given explicitly. In other words, $M_{\mathrm{Sym}(10)}^{0}\left(\Gamma^{(2)}\right)$ is generated by 13 modular forms of determinant weights 6,8 , $10,10,12,12,14,14,14,16,16,18,20$ and they satisfy two fundamental relations. $M_{\mathrm{Sym}(10)}^{1}\left(\Gamma^{(2)}\right)$ is generated by 13 modular forms of determinant weights $9,11,13$, $15,15,15,17,17,17,19,19,21,23$ and they satisfy two fundamental relations.

In $\$ 6$, we give relations and generators explicitly.

By Igusa [10, there exists a cusp form $\chi_{35} \in M_{35}\left(\Gamma^{(2)}\right)$ of weight 35 and we have

$$
M_{\text {Sym }(0)}^{1}\left(\Gamma^{(2)}\right)=A_{\text {ev }} \chi_{35} .
$$

And by Igusa [9], $A_{\mathrm{ev}}$ is isomorphic to the polynomial ring of 4 variables over $\mathbb{C}$ and is generated by $\phi_{4}, \phi_{6}, \chi_{10}$ and $\chi_{12}$. Here $\phi_{4}$ (resp. $\phi_{6}$ ) is the Siegel Eisenstein series of weight 4 (resp. 6). And $\chi_{10}$ (resp. $\chi_{12}$ ) is the cusp form of weight 10 (resp. 12). We put $K_{\mathrm{ev}}=\operatorname{Frac}\left(A_{\mathrm{ev}}\right)$. Let $j \in 2 \mathbb{Z}_{\geq 0}$ and $\epsilon=0$ or 1 . By 20 , we have

$$
\operatorname{dim}_{K_{\mathrm{ev}}} M_{\mathrm{Sym}(j)}^{\epsilon}\left(\Gamma^{(2)}\right) \otimes_{A_{\mathrm{ev}}} K_{\mathrm{ev}}=j+1 .
$$

We put

$$
\operatorname{det}\left(M_{\operatorname{Sym}(j)}^{\epsilon}\left(\Gamma^{(2)}\right)\right)=\wedge^{j+1} M_{\operatorname{Sym}(j)}^{\epsilon}\left(\Gamma^{(2)}\right),
$$

then $\operatorname{det}\left(M_{\operatorname{Sym}(j)}^{\epsilon}\left(\Gamma^{(2)}\right)\right) \neq 0$. We fix an isomorphism $\wedge^{j+1} \operatorname{Sym}(j) \cong \operatorname{det}^{j(j+1) / 2}$. Then for $f_{i} \in M_{k_{i}, j}\left(\Gamma^{(2)}\right)(1 \leq i \leq j+1)$, we have $f_{1} \wedge \cdots \wedge f_{j+1} \in M_{k}\left(\Gamma^{(2)}\right)$, where $k=\sum_{i} k_{i}+j(j+1) / 2$. Since $j$ is even, we have

$$
\operatorname{det}\left(M_{\mathrm{Sym}(j)}^{\epsilon}\left(\Gamma^{(2)}\right)\right) \subset \begin{cases}A_{\mathrm{ev}} & \text { if } j / 2+\epsilon \text { is even, } \\ A_{\mathrm{ev}} \chi_{35} & \text { if } j / 2+\epsilon \text { is odd }\end{cases}
$$

Thus there exists a non-zero ideal $I_{j, \epsilon}$ of $A_{\text {ev }}$ such that

$$
\operatorname{det}\left(M_{\operatorname{Sym}(j)}^{\epsilon}\left(\Gamma^{(2)}\right)\right)= \begin{cases}I_{j, \epsilon} & \text { if } j / 2+\epsilon \text { is even } \\ I_{j, \epsilon} \chi_{35} & \text { if } j / 2+\epsilon \text { is odd }\end{cases}
$$

Definition 1.1. For $j \in 2 \mathbb{Z}_{\geq 0}$ and $\epsilon=0,1$, we denote by $\operatorname{gcd}\left(I_{j, \epsilon}\right)$ the greatest common divisor of generators of the ideal $I_{j, \epsilon}$ above. We define $f_{j, \epsilon} \in A_{\mathrm{ev}} \oplus A_{\mathrm{ev}} \chi_{35}$ by

$$
f_{j, \epsilon}= \begin{cases}\operatorname{gcd}\left(I_{j, \epsilon}\right) & \text { if } j / 2+\epsilon \text { is even, } \\ \operatorname{gcd}\left(I_{j, \epsilon}\right) \chi_{35} & \text { if } j / 2+\epsilon \text { is odd }\end{cases}
$$


The element $f_{j, \epsilon}$ is defined up to a non-zero constant.

\section{Remark 1.1.}

(1) In $\$ 4$, we construct $f_{j, \epsilon}$ by the alternating product of minors of matrices appearing in a free resolution of $M_{\operatorname{Sym}(j)}^{\epsilon}\left(\Gamma^{(2)}\right)$.

(2) If $M_{\operatorname{Sym}(j)}^{\epsilon}\left(\Gamma^{(2)}\right)$ is a free $A_{\text {ev }}$ module and $\left\{f_{1}, \ldots, f_{j+1}\right\}$ is its basis, we can take $f_{j, \epsilon}$ as $f_{1} \wedge \cdots \wedge f_{j+1}$.

(3) By structure theorems of $M_{\mathrm{Sym}(2)}^{\epsilon}\left(\Gamma^{(2)}\right)$ (cf. [17, [7]) and the result on a Wronskian of scalar valued Siegel modular forms (cf. [1]), we have

$$
\begin{aligned}
& \operatorname{det}\left(M_{\operatorname{Sym}(2)}^{0}\left(\Gamma^{(2)}\right)\right)=\left(\phi_{4}, \phi_{6}, \chi_{10}, \chi_{12}\right)^{2} \chi_{35}, \\
& \operatorname{det}\left(M_{\operatorname{Sym}(2)}^{1}\left(\Gamma^{(2)}\right)\right)=\left(\phi_{4}, \phi_{6}, \chi_{10}, \chi_{12}\right) \chi_{35}^{2} .
\end{aligned}
$$

Thus we have $f_{2,0}=\chi_{35}$ and $f_{2,1}=\chi_{35}^{2}$ up to non-zero constants.

In \$4, we shall prove

Proposition 1.1. For $j \in 2 \mathbb{Z}_{\geq 0}$ and $\epsilon=0,1$, we have

$$
f_{j, \epsilon} \in M_{35(j / 2+\epsilon)}\left(\Gamma^{(2)}\right) \text {. }
$$

The second main result of this paper is the following theorem.

Theorem 1.2. Let $j$ be an even number and assume $0 \leq j \leq 10$. For $\epsilon=0,1$, we have

$$
f_{j, \epsilon}=\chi_{35}^{j / 2+\epsilon}
$$

up to a non-zero constant.

Remark 1.2. The statement of the theorem was conjectured by Ibukiyama [7] if $j=4,6,8$.

We recall preceding results on the structure theorems of the graded $A_{\mathrm{ev}}$ module $M_{\mathrm{Sym}(j)}^{\epsilon}\left(\Gamma^{(2)}\right)$. First, Igusa [9], 10] gave generators of $A_{\mathrm{ev}}=M_{\mathrm{Sym}(0)}^{0}\left(\Gamma^{(2)}\right)$ and proved that $M_{\mathrm{Sym}(0)}^{1}\left(\Gamma^{(2)}\right)$ is a free $A_{\mathrm{ev}}$ module of rank 1. T. Satoh 17 proved the structure theorem of $M_{\mathrm{Sym}(2)}^{0}\left(\Gamma^{(2)}\right)$. The module $M_{\mathrm{Sym}(2)}^{0}\left(\Gamma^{(2)}\right)$ is generated by six modular forms, they satisfy four relations and the four relations satisfy one relation. Ibukiyama 7$]$ proved structure theorems of $M_{\mathrm{Sym}(2)}^{1}\left(\Gamma^{(2)}\right), M_{\mathrm{Sym}(4)}^{0}\left(\Gamma^{(2)}\right)$, $M_{\mathrm{Sym}(4)}^{1}\left(\Gamma^{(2)}\right)$ and $M_{\mathrm{Sym}(6)}^{0}\left(\Gamma^{(2)}\right)$. The module $M_{\mathrm{Sym} 2}^{1}\left(\Gamma^{(2)}\right)$ is generated by four modular forms and they satisfy one relation. $M_{\operatorname{Sym}(j)}^{\epsilon}\left(\Gamma^{(2)}\right)$ is free of rank $j+1$ for $(j, \epsilon)=(4,0),(4,1),(6,0)$. Van Dorp 22 proved $M_{\operatorname{Sym}(6)}^{1}\left(\Gamma^{(2)}\right)$ is free of rank 7 . Kiyuna 13 proved $M_{\operatorname{Sym}(8)}^{\epsilon}\left(\Gamma^{(2)}\right)$ is free of rank 9 for $\epsilon=0,1$. And they all gave generators and relations explicitly.

By Theorem 1.1 and $\$ 6$, we can compute a basis of $M_{k, 10}\left(\Gamma^{(2)}\right)$ explicitly. This is one of motivations of this paper. In $\$ 8$, we give some examples of Hecke eigenforms. A program for computing $M_{k, 10}\left(\Gamma^{(2)}\right)$ can be found at 19$]$. 


\section{Definition and notation}

In this section, we define Siegel modular forms of degree two and introduce notation used throughout in this paper. We define Siegel upper half space $\mathbb{H}_{2}$ of degree two by

$$
\mathbb{H}_{2}=\left\{x+i y \mid x, y \in \operatorname{Sym}_{2}(\mathbb{R}), y \text { is positive definite }\right\} .
$$

Here $\operatorname{Sym}_{2}(\mathbb{R})$ is the space of symmetric matrices with entries in $\mathbb{R}$. For a commutative ring $R$, we define the symplectic group of degree 2 by

$$
\mathrm{Sp}_{2}(R)=\left\{g \in \mathrm{GL}_{4}(R) \mid{ }^{t} g w_{2} g=w_{2}\right\}
$$

where $w_{2}=\left(\begin{array}{cc}0_{2} & -1_{2} \\ 1_{2} & 0_{2}\end{array}\right)$. Then $\operatorname{Sp}_{2}(\mathbb{R})$ acts on $\mathbb{H}_{2}$ by

$$
\left(\begin{array}{ll}
a & b \\
c & d
\end{array}\right) \cdot z=(a z+b)(c z+d)^{-1}, \quad\left(\begin{array}{ll}
a & b \\
c & d
\end{array}\right) \in \mathrm{Sp}_{2}(\mathbb{R}), z \in \mathbb{H}_{2} .
$$

Here $a, b, c, d \in M_{2}(\mathbb{R})$. We put $\Gamma^{(2)}=\mathrm{Sp}_{2}(\mathbb{Z})$.

Let $\rho: \mathrm{GL}_{2}(\mathbb{C}) \rightarrow \operatorname{Aut}_{\mathbb{C}}(V)$ be an irreducible polynomial representation of $\mathrm{GL}_{2}(\mathbb{C})$ and $\chi: \mathrm{Sp}_{2}(\mathbb{Z}) \rightarrow \mathbb{C}^{\times}$a character. A $V$-valued holomorphic function $f$ : $\mathbb{H}_{2} \rightarrow V$ is said to be a (holomorphic) Siegel modular form of degree two, weight $\rho$ and character $\chi$ if

$$
f(g \cdot z)=\rho(c z+d) \chi(g) f(z), \quad \text { for all } g=\left(\begin{array}{ll}
a & b \\
c & d
\end{array}\right) \in \operatorname{Sp}_{2}(\mathbb{Z}) .
$$

We denote the space of Siegel modular forms of degree two, weight $\rho$ and character $\chi$ by $M_{\rho}\left(\Gamma^{(2)}, \chi\right)$. If $\chi$ is the trivial character, we simply denote $M_{\rho}\left(\Gamma^{(2)}, \chi\right)$ by $M_{\rho}\left(\Gamma^{(2)}\right)$.

For a non-negative integer $j$, we denote by $V_{j}$ the space of homogeneous polynomials of $u_{1}$ and $u_{2}$ of degree $j$, where $u_{1}$ and $u_{2}$ are variables. We define the symmetric representation $\operatorname{Sym}(j)$ of degree $j$ by

$$
(\operatorname{Sym}(j)(\alpha) P)\left(u_{1} u_{2}\right)=P\left(\left(u_{1} u_{2}\right) \alpha\right),
$$

for $P \in V_{j}$ and $\alpha \in \mathrm{GL}_{2}(\mathbb{C})$. We denote by det the determinant representation of $\mathrm{GL}_{2}(\mathbb{C})$. When $\rho=\operatorname{det}^{k} \otimes \operatorname{Sym}(j)$, we put $M_{k, j}\left(\Gamma^{(2)}\right)=M_{\rho}\left(\Gamma^{(2)}\right)$. For $F \in$ $M_{k, j}\left(\Gamma^{(2)}\right)$, we define the Siegel operator by

$$
\Phi(F)(z)=\lim _{t \rightarrow+\infty} F\left(\left(\begin{array}{cc}
z & 0 \\
0 & i t
\end{array}\right)\right) .
$$

Then by Arakawa [2], we have

$$
\Phi(F) \in S_{k+j}\left(\mathrm{SL}_{2}(\mathbb{Z})\right) u_{1}^{j},
$$

if $j>0$. Here $S_{k+j}\left(\mathrm{SL}_{2}(\mathbb{Z})\right)$ is the space of elliptic cusp forms of weight $k+j$, level 1. For $F \in M_{k, j}\left(\Gamma^{(2)}\right)$, we call $F$ a cusp form if $\Phi(F)=0$ and denote by $S_{k, j}\left(\Gamma^{(2)}\right)$ the space of cusp forms. 
Let $F \in M_{k, j}\left(\Gamma^{(2)}\right)$. Then by Köcherer principle, we have the following Fourier expansion:

$$
F\left(\left(\begin{array}{ll}
z_{11} & z_{12} \\
z_{12} & z_{22}
\end{array}\right)\right)=\sum_{n, r, m} a((n, r, m) ; F) \exp \left(2 \pi\left(n z_{11}+r z_{12}+m z_{22}\right)\right) .
$$

Here $a((n, r, m) ; F) \in V_{j}$ and the summation index runs over the following set:

$$
\left\{(n, r, m) \mid n, r, m \in \mathbb{Z}, n, m, 4 n m-r^{2} \geq 0\right\} .
$$

Let $q_{11}, q_{12}, q_{22}$ be variables and $\mathbb{C}\left[q_{12}, q_{12}^{-1}\right]\left[q_{11}, q_{22} \rrbracket\right.$ a ring of formal power series. We embed $M_{k, j}\left(\Gamma^{(2)}\right)$ to $\mathbb{C}\left[q_{12}, q_{12}^{-1}\right]\left[q_{11}, q_{22} \rrbracket \otimes_{\mathbb{C}} V_{j}\right.$ by

$$
F \mapsto \sum_{i=0}^{j}\left(\sum_{n, r, m} a((n, r, m) ; F)_{i} q_{11}^{n} q_{12}^{r} q_{22}^{m}\right) u_{1}^{j-i} u_{2}^{i},
$$

where for $v \in V_{j}$, we denote by $v_{i}$ the coefficient of $u_{1}^{j-i} u_{2}^{i}$ of $v$.

\section{Generators of the ring of scalar valued modular forms}

Later, we construct vector valued Siegel modular forms by scalar valued Siegel modular forms. We recall well-known results on the generator of the ring of scalar valued modular forms of degree two.

For an even integer $k \geq 4$, we denote by $\phi_{k}$ the Siegel Eisenstein series of degree two and weight $k$. We normalize $\phi_{k}$ so that $a\left((0,0,0) ; \phi_{k}\right)=1$. We put

$$
\begin{gathered}
\chi_{10}=\frac{2^{10} \cdot 3^{5} \cdot 5^{2} \cdot 7 \cdot 53}{43867}\left(\phi_{4} \phi_{6}-\phi_{10}\right), \\
\chi_{12}=\frac{2^{11} \cdot 3^{6} \cdot 5^{3} \cdot 7^{2} \cdot 337}{131 \cdot 593}\left(3^{2} \cdot 7^{2} \phi_{4}^{3}+2 \cdot 5^{3} \phi_{6}^{2}-691 \phi_{12}\right) .
\end{gathered}
$$

Then we have $\chi_{k} \in S_{k}\left(\Gamma^{(2)}\right)$ and $a\left((1,1,1) ; \chi_{k}\right)=1$ for $k=10,12$. We can also construct $\chi_{10}$ and $\chi_{12}$ by the Saito-Kurokawa lift.

Theorem 3.1 (Igusa [9], [10]). The modular forms $\phi_{4}, \phi_{6}, \chi_{10}$ and $\chi_{12}$ are algebraically independent over $\mathbb{C}$ and we have

$$
A_{\mathrm{ev}}=\mathbb{C}\left[\phi_{4}, \phi_{6}, \chi_{10}, \chi_{12}\right], \quad M_{\mathrm{Sym}(0)}^{1}\left(\Gamma^{(2)}\right)=\chi_{35} A_{\mathrm{ev}},
$$

where $\chi_{35} \in S_{35}\left(\Gamma^{(2)}\right)$ is a cusp form of weight 35 .

Let sgn be the unique nontrivial character of $\Gamma^{(2)}$ (see [14]). Then sgn is quadratic. There exists a square root $\chi_{5} \in S_{5}\left(\Gamma^{(2)}, \operatorname{sgn}\right)$ of $\chi_{10}$. The cusp form $\chi_{5}$ has the following Fourier expansion:

$$
\chi_{5}(Z)=\sum_{\substack{n, m, m, 4 n m-r^{2} \geq 0 \\ n, r, m \in 1 / 2+\mathbb{Z}}} a\left((n, r, m) ; \chi_{5}\right) \mathbf{e}\left(n z_{11}+r z_{12}+m z_{22}\right) .
$$

We normalize $\chi_{5}$ so that $a\left((1 / 2,1 / 2,1 / 2) ; \chi_{5}\right)=1$. 
Fourier coefficients of Siegel-Eisenstein series $\phi_{k}$ are explicitly known and the computation is not difficult. We can compute Fourier coefficients of $\chi_{35}$ by the Wronskian given in [1] . Since $\chi_{5}$ is the Saito-Kurokawa lift of a Jacobi theta series (see [15, 6]), we can easily compute Fourier coefficients of $\chi_{5}$.

\section{The determinant of the module of vector valued Siegel modular forms}

In this section, we give an another interpretation of $f_{j, \epsilon}$ in Definition 1.1 and prove Proposition 1.1

\subsection{The determinant of a based exact sequence}

We recall the definition and basic property of the determinant of a based exact sequence over a field. We follow appendix A of [5].

Let $K$ be a field and $W$ a vector space of dimension $n<\infty$ over $K$. We put $\operatorname{det}(W)=\wedge^{n} W$. For a one dimensional vector space $V$, we put $V^{-1}=\operatorname{Hom}_{K}(V, K)$. For a non-zero element $v \in V$, we denote by $v^{-1}$ the element of $V^{-1}$ such that $v^{-1}(v)=1$.

For $i \in \mathbb{Z}$, let $W_{i}$ be a finite dimensional vector space over a field $K$ and $d_{i}$ : $W_{i} \rightarrow W_{i+1}$ a linear map. We put $d=\left\{d_{i} \mid i \in \mathbb{Z}\right\}$ and $W_{\bullet}=\bigoplus_{i \in \mathbb{Z}} W_{i}$. We assume $\left(W_{\bullet}, d\right)$ forms a finite exact sequence, that is there exists $n \in \mathbb{Z}_{\geq 0}$ such that $W_{i}=0$ for $|i| \geq n$ and $\operatorname{ker}\left(d_{i}\right)=\operatorname{Im}\left(d_{i-1}\right)$ for all $i$. We define the determinant of $W \bullet$ by

$$
\operatorname{det}\left(W_{\bullet}\right)=\bigotimes_{i} \operatorname{det}\left(W_{i}\right)^{(-1)^{i}} .
$$

Then by [5], there is a natural isomorphism called the Euler isomorphism

$$
\mathrm{Eu}_{d}: \operatorname{det}\left(W_{\bullet}\right) \rightarrow K .
$$

For a three term exact sequence

$$
0 \rightarrow W_{0} \stackrel{d_{0}}{\longrightarrow} W_{1} \stackrel{d_{1}}{\longrightarrow} W_{2} \rightarrow 0,
$$

the Euler isomorphism $\operatorname{det}\left(W_{0}\right) \otimes \operatorname{det}\left(W_{2}\right) \otimes\left(\operatorname{det}\left(W_{1}\right)\right)^{-1} \rightarrow K$ is given as follows. Suppose $a_{1} \wedge \cdots \wedge a_{n} \in \operatorname{det}\left(W_{0}\right), c_{1} \wedge \cdots \wedge c_{m} \in \operatorname{det}\left(W_{2}\right)$ and $f \in$ $\left(\operatorname{det}\left(W_{1}\right)\right)^{-1}$. For $c_{j} \in W_{2}$, we take $\widetilde{c}_{j} \in W_{1}$ such that $d_{1}\left(\widetilde{c}_{j}\right)=c_{j}$. Then we define $\mathrm{Eu}_{d}\left(a_{1} \wedge \cdots \wedge a_{n} \wedge c_{1} \cdots \wedge c_{m} \wedge f\right)$ by

$$
f\left(d_{0}\left(a_{1}\right) \wedge \cdots \wedge d_{0}\left(a_{n}\right) \wedge \widetilde{c}_{1} \wedge \cdots \wedge \widetilde{c}_{m}\right) .
$$

It is easy to see that this is well-defined. The definition for general exact sequences is reduced to this case.

Let $W$ be a vector space and $e=\left\{e_{\alpha} \mid 1 \leq \alpha \leq \operatorname{dim}_{K}(W)\right\}$ a basis of $W$. Denote by $\operatorname{det}(e)$ by the wedge product $e_{1} \wedge \cdots \wedge e_{\operatorname{dim}_{K}(W)}$. Let $\left(W_{\bullet}, d\right)$ be a finite exact 
sequence and suppose that $e=\{e(i)\}$ is a system of bases in all $W_{i}$ so that each $e(i)$ is a basis in $W_{i}$. We $\operatorname{define} \operatorname{det}(e) \in \operatorname{det}\left(W_{\bullet}\right)$ by

$$
\operatorname{det}(e)=\bigotimes_{i} \operatorname{det}(e(i))^{(-1)^{i}}
$$

Here we put $\operatorname{det}(e(i))=1$ if $W_{i}=\{0\}$. We call $\left(W_{\bullet}, d, e\right)$ a based exact sequence.

Definition 4.1. For a based exact sequence $\left(W_{\bullet}, d, e\right)$, we define its determinant by

$$
\operatorname{det}\left(W_{\bullet}, d, e\right)=\mathrm{Eu}_{d}(\operatorname{det}(e)) \in K^{\times} .
$$

Next we explain how to compute determinants of based exact sequences. Let $\left(W_{\bullet}, d, e\right)$ be a based exact sequence. For $i \in \mathbb{Z}$, let $B_{i}=\left\{1 \leq \alpha \leq \operatorname{dim}_{K} W_{i}\right\}$ be an ordered set of indices of basis. Assume $d_{i} \neq 0$. We denote by $D_{i}$ the matrix representation of $d_{i}$ with respect to $e(i)$ and $e(i+1)$. We put $D_{i}=\left(m_{\alpha, \beta}^{(i)}\right)_{\alpha \in B_{i+1}, \beta \in B_{i}}$. For subsets $X \subset B_{i+1}$ and $Y \subset B_{i}$, we define $\left(D_{i}\right)_{X, Y}$ by the sub-matrix $\left(m_{\alpha, \beta}^{(i)}\right)_{\alpha \in X, \beta \in Y}$.

Definition 4.2. A collection of subsets $I_{i} \subset B_{i}(i \in \mathbb{Z})$ is called admissible if

$$
\left|I_{i}\right|=\sum_{j \geq 0}(-1)^{j} \operatorname{dim}_{K} W_{i-1-j}
$$

and $\left(D_{i}\right)_{I_{i+1}, B_{i} \backslash I_{i}}$ is invertible if $d_{i} \neq 0$.

Remark 4.1. It can be easily proved that an admissible collection exists (see [5] ).

We can compute the determinant of a based exact sequence by the alternating product of minors. For the proof of the following theorem, see [5] .

Theorem 4.1. Let $\left(I_{i}\right)$ be an admissible collection for a based exact sequence $\left(W_{\bullet}, d, e\right)$. We put $\Delta_{i}=\operatorname{det}\left(\left(D_{i}\right)_{I_{i+1}, B_{i} \backslash I_{i}}\right)$. Then we have

$$
\operatorname{det}\left(W_{\bullet}, d, e\right)=\prod_{i \in \mathbb{Z}} \Delta_{i}^{(-1)^{i-1}} .
$$

Here we understand $\Delta_{i}=1$ if $d_{i}=0$.

Example 4.1. Let $n \in \mathbb{Z}_{\geq 1}$. We define a three term based exact sequence as follows.

$$
0 \rightarrow W_{-2} \stackrel{d_{-2}}{\longrightarrow} W_{-1} \stackrel{d_{-1}}{\longrightarrow} W_{0} \rightarrow 0
$$

where $W_{-2}=K, W_{-1}=K^{n+1}, W_{0}=K^{n}$. We take a basis $e(i)$ of $W_{i}$ by the standard basis of $W_{i}$. Let ${ }^{t}\left(a_{1}, a_{2}, \ldots, a_{n+1}\right)$ be the matrix representation of $d_{-2}$ with respect to the standard bases, $e_{1}, e_{2}, \ldots, e_{n+1}$ the standard basis of $W_{-1}$ and 
$f_{1}, f_{2}, \ldots, f_{n}$ the standard basis of $W_{0}$. Let $B_{i}=\left\{1 \leq \alpha \leq \operatorname{dim}_{K} W_{i}\right\}$ be the ordered set of indices of basis. We assume $a_{n+1} \neq 0$. We define a subset $I_{i} \subset B_{i}$ by

$$
I_{i}= \begin{cases}B_{i} & \text { if } i \geq 0, \\ \{n+1\} & \text { if } i=-1, \\ \emptyset & \text { if } i \leq-2 .\end{cases}
$$

Then $I_{i}$ is an admissible collection. Let $\Delta_{i}$ be the determinant defined in Theorem 4.1. Then we have

$$
\begin{gathered}
\Delta_{-1} f_{1} \wedge f_{2} \wedge \cdots \wedge f_{n}=d_{-1}\left(e_{1}\right) \wedge d_{-1}\left(e_{2}\right) \wedge \cdots \wedge d_{-1}\left(e_{n}\right), \\
\Delta_{-2}=a_{n+1} .
\end{gathered}
$$

Thus by Theorem 4.1 .

$$
\operatorname{det}\left(W_{\bullet}, d, e\right) f_{1} \wedge f_{2} \wedge \cdots \wedge f_{n}=a_{n+1}^{-1} d_{-1}\left(e_{1}\right) \wedge d_{1}\left(e_{2}\right) \wedge \cdots \wedge d_{-1}\left(e_{n}\right),
$$

where $\operatorname{det}\left(W_{\bullet}, d, e\right)$ is the determinant of 4.1 .

\subsection{The determinant of a free resolution of $M_{\mathrm{Sym}(j)}^{\epsilon}\left(\Gamma^{(2)}\right)$}

Next, we define the determinant of a given free resolution of $M_{\operatorname{Sym}(j)}^{\epsilon}\left(\Gamma^{(2)}\right)$ for $\epsilon=0,1$. And we shall prove that this determinant satisfies the property given in Definition 1.1.

Definition 4.3. Let $\epsilon=0,1$ and

$$
0 \rightarrow F_{-r} \stackrel{\psi_{-r}}{\longrightarrow} F_{-r+1} \stackrel{\psi_{-r+1}}{\longrightarrow} \cdots \stackrel{\psi_{-2}}{\longrightarrow} F_{-1} \stackrel{\psi_{-1}}{\longrightarrow} M \rightarrow 0
$$

a free resolution of $M=M_{\operatorname{Sym}(j)}^{\epsilon}\left(\Gamma^{(2)}\right)$ as a graded $A_{\mathrm{ev}}$ module. Here

$$
F_{i}=\bigoplus_{1 \leq \nu \leq n_{i}} A_{\mathrm{ev}}\left(-a_{\nu}^{(i)}\right), \quad \text { for }-r \leq i \leq-1
$$

be a graded free module, where $a_{\nu}^{(i)} \in \mathbb{Z}$. We take a basis $e(0)=\left\{f_{1}, \ldots, f_{j+1}\right\}$ of $M$. Define a based exact sequence $\left(W_{\bullet}, d, e\right)$ associated with the free resolution Rsl and $e(0)$ as follows. We put $W_{0}=M \otimes_{A_{\mathrm{ev}}} K_{\mathrm{ev}}$ and $W_{i}=F_{i} \otimes_{A_{\mathrm{ev}}} K_{\mathrm{ev}}$ for $-r \leq i \leq-1$. For $i>0$ or $i<-r$, we put $W_{i}=0$. We take a basis $e(i)$ of $W_{i}$ as the basis obtained by the standard basis of $F_{i}$. We define $d_{i}: W_{i} \rightarrow W_{i+1}$ by $d_{i}=\psi_{i} \otimes_{A_{\mathrm{ev}}} \mathrm{id}_{K_{\mathrm{ev}}}$. Then we define the determinant of the free resolution Rsl by

$$
\operatorname{det}(\operatorname{Rsl})=\operatorname{det}\left(W_{\bullet}, d, e\right) f_{1} \wedge \cdots \wedge f_{j+1} .
$$

By [5], $\operatorname{det}(\mathrm{Rsl})$ does not depend on the choice of the basis $f_{1}, \ldots, f_{j+1}$.

Proposition 4.1. For $j \in 2 \mathbb{Z}_{\geq 0}$ and $\epsilon=0,1$, let $\operatorname{det}(\mathrm{Rsl})$ be the determinant given in Definition 4.3 and $f_{j, \epsilon}$ an element of $A_{\mathrm{ev}} \oplus A_{\mathrm{ev}} \chi_{35}$ given in Definition 1.1. Then we have

$$
\operatorname{det}(\operatorname{Rsl})=f_{j, \epsilon},
$$


up to a non-zero constant.

Proof. By definition of $f_{j, \epsilon}$, it is enough to prove

$$
A_{P} \operatorname{det}(\mathrm{Rsl})=\operatorname{det}(M) \otimes_{A} A_{P},
$$

for any height one prime $P$ of $A$. Here $M=M_{\mathrm{Sym}(j)}^{\epsilon}\left(\Gamma^{(2)}\right)$ and $A=A_{\mathrm{ev}}$. In the proof, we use the same notation in Definition 4.3 Let $P$ be a height one prime of $A$. Since $A_{P}$ is a discrete valuation ring, we can take a basis $\left\{f_{1}, \ldots, f_{j+1}\right\}$ of $M$ so that $\operatorname{det}(M) \otimes_{A} A_{P}=A_{P} f_{1} \wedge \cdots \wedge f_{j+1}$. Put $\kappa=A_{P} / P A_{P}$. Since $M$ and $F_{i}$ $(-r \leq i \leq-1)$ are torsion free $A$ modules, the following sequence $\operatorname{Rsl} \otimes_{A} \kappa$ is exact.

$$
0 \rightarrow F_{-r} \otimes_{A} \kappa \rightarrow F_{-r+1} \otimes_{A} \kappa \cdots \rightarrow F_{-1} \otimes_{A} \kappa \rightarrow M \otimes_{A} \kappa \rightarrow 0
$$

We define a based exact sequence $\left(V_{\bullet}, \bar{d}, \bar{e}\right)$ over $\kappa$ as follows. We put $V_{0}=M \otimes_{A} \kappa$ and $V_{i}=F_{i} \otimes_{A} \kappa$ for $-r \leq i \leq-1$. Otherwise we put $V_{i}=0$. Define $\bar{d}_{i}: V_{i} \rightarrow V_{i+1}$ by $\bar{d}_{i}=\psi_{i} \otimes{ }_{A} \operatorname{id}_{\kappa}$. Let $\bar{e}(0)=\left\{f_{1} \otimes 1_{\kappa}, \cdots, f_{j+1} \otimes 1_{\kappa}\right\}$ and $\bar{e}(i)$ a basis obtained by the standard basis of $F_{i}$ for $-r \leq i \leq-1$. Denote by $B_{i}$ the ordered index set of $\bar{e}(i)$. We take an admissible collection $\left\{I_{i}\right\}$ for a based exact sequence $\left(V_{\bullet}, \bar{d}, \bar{e}\right)$. Then $\left\{I_{i}\right\}$ is an admissible collection for $\left(W_{\bullet}, d, e\right)$ in Definition 4.3 Then by Theorem 4.1 we have $\operatorname{det}\left(W_{\bullet}, d, e\right) \in A_{P}^{\times}$. Therefore we have $A_{P} \operatorname{det}(\mathrm{Rsl})=A_{P} f_{1} \wedge \cdots \wedge f_{j+1}=$ $\operatorname{det}(M) \otimes_{A} A_{P}$.

Since Rsl is a free resolution as a graded module, there exist $f \in M_{k}\left(\Gamma^{(2)}\right)$ and $g \in M_{l}\left(\Gamma^{(2)}\right)$ for some $k$ and $l$ such that $\operatorname{det}(\mathrm{Rsl})$ is equal to $f / g$. By Proposition 4.1 , we can take $g=1$. We can compute the weight of $f$.

Proposition 4.2. Let $\operatorname{det}(\mathrm{Rsl})$ be the determinant given in Definition 4.3. Then the weight of $\operatorname{det}(\mathrm{Rsl})$ is equal to $35(j / 2+\varepsilon)$.

Proof. Let $\left(W_{\bullet}, d, e\right)$ be a based exact sequence given in Definition $4.3, D_{i}$ the matrix representation of $d_{i}$ with respect to $e(i)$ and $e(i+1)$ and $B_{i}$ the ordered index set of $e(i)$. We take an admissible collection $\left\{I_{i}\right\}$ for $\left(W_{\bullet}, d, e\right)$. For $f \in M_{k}\left(\Gamma^{(2)}\right)$, we denote by $\operatorname{wt}(f)$ the weight of $f$. For $-r \leq i \leq-2$ and $\alpha \in B_{i+1}, \beta \in B_{i}$, the weight of $(\alpha, \beta)$-entry of $D_{i}$ is equal to $a_{\beta}^{(i)}-a_{\alpha}^{(i+1)}$. Therefore we have

$$
\operatorname{wt}\left(\left(\Delta_{i}\right)_{I_{i+1}, B_{i} \backslash I_{i}}\right)=\sum_{\alpha \in B_{i} \backslash I_{i}} a_{\alpha}^{(i)}-\sum_{\alpha \in I_{i+1}} a_{\alpha}^{(i+1)},
$$

for $-r \leq i \leq-2$. By Theorem 4.1 , we have

$$
\operatorname{wt}\left(\operatorname{det}\left(\left(W_{\bullet}, d, e\right)\right)\right)=\sum_{-r \leq i \leq-2}(-1)^{i-1} \sum_{\alpha \in B_{i}} a_{\alpha}^{(i)}+\sum_{\alpha \in I_{-1}} a_{\alpha}^{(-1)} \text {. }
$$

By

$$
\operatorname{wt}\left(f_{1} \wedge \cdots \wedge f_{j+1}\right)=\sum_{\alpha \in B_{-1} \backslash I_{-1}} a_{\alpha}^{(-1)}+\frac{1}{2} j(j+1),
$$


we have

$$
\operatorname{wt}(\operatorname{det}(\mathrm{Rsl}))=\sum_{-r \leq i \leq-1}(-1)^{i-1} \sum_{\alpha \in B_{i}} a_{\alpha}^{(i)}+\frac{1}{2} j(j+1) .
$$

Therefore we obtain the assertion of our Proposition by [20].

Corollary 4.1. Proposition 1.1 is true.

Proof. This follows from Proposition 4.1 and Proposition 4.2

Next we compute $f_{j, \epsilon}$ given in Definition 1.1 for $j \leq 8$.

Proposition 4.3. For $j=0,2,4,6,8$ and $\epsilon=0,1$, let $f_{j, \epsilon}$ be a Siegel modular form given in Definition 1.1. Then we have

$$
f_{j, \epsilon}=\chi_{35}^{j / 2+\epsilon}
$$

up to a non-zero constant.

Proof. If $j=0$, the assertion follows from the structure theorem proved by Igusa [9], [10. If $j=4,6$, the assertion follows from 20], since $M_{\operatorname{Sym}(j)}^{\epsilon}\left(\Gamma^{(2)}\right)$ is free in this case. We can check the assertion in the case when $j=8$ by a similar computation to [20]. Source code, for checking this, can be found at https://github.com/stakemori/det_vec_vald_SMFs. Suppose $j=2$. For simplicity, we assume $\epsilon=1$. By Ibukiyama $[7], M=M_{\operatorname{Sym}(2)}^{1}\left(\Gamma^{(2)}\right)$ has the following free resolution.

$$
0 \rightarrow A(-33) \rightarrow A(-21) \oplus A(-23) \oplus A(-27) \oplus A(-29) \rightarrow M \rightarrow 0
$$

Here $A=A_{\mathrm{ev}}$. In the matrix form, the second map is given by

$$
{ }^{t}\left(-12 \chi_{12}, 10 \chi_{10},-6 \phi_{6}, 4 \phi_{4}\right) .
$$

The third map sends the standard basis to elements defined in 7 of determinant weights $21,23,27,29$ respectively. We write them as $g_{21}, g_{23}, g_{27}$ and $g_{29}$. We use the same notation in Definition 4.3 and put $f_{1}=g_{21}, f_{2}=g_{23}, f_{3}=g_{27}$. We define $I_{i} \subset B_{i}$ by

$$
I_{i}= \begin{cases}B_{i} & \text { if } i \geq 0 \\ \{4\} & \text { if } i=-1 \\ \emptyset & \text { if } i \leq-2\end{cases}
$$

Then $\left\{I_{i}\right\}$ is an admissible collection. By Theorem 4.1, we have

$$
\operatorname{det}\left(\left(W_{\bullet}, d, e\right)\right)=\left(4 \phi_{4}\right)^{-1} \text {. }
$$

By the proof of $[7]$, we have

$$
g_{21} \wedge g_{23} \wedge g_{27}=\phi_{4} \chi_{35}^{2},
$$

up to a non-zero constant. By definition, the determinant of the free resolution is equal to $4^{-1} \chi_{35}^{2}$. By Proposition 4.1. we have our assertion. 


\section{Rankin-Cohen-Ibukiyama type differential operators}

We use Rankin-Cohen-Ibukiyama type differential operators to construct generators of $M_{\text {Sym }(10)}^{0}\left(\Gamma^{(2)}\right)$ and $M_{\text {Sym(10) }}^{1}\left(\Gamma^{(2)}\right)$. We recall differential operators defined by Eholzer-Ibukiyama [3] and van Dorp [22. We use the same notation used in [11].

Let $k, l$ be positive integers and $\chi, \psi$ characters of $\Gamma^{(2)}$. We take scalar valued Siegel modular forms $f \in M_{k}\left(\Gamma^{(2)}, \chi\right)$ and $g \in M_{l}\left(\Gamma^{(2)}, \psi\right)$. For $j \in \mathbb{Z}_{\geq 0}$, EholzerIbukiyama [3] constructed vector valued Siegel modular forms

$$
\begin{aligned}
& \{f, g\}_{\operatorname{Sym}(j)} \in M_{k+l, j}\left(\Gamma^{(2)}, \chi \psi\right), \\
& \{f, g\}_{\operatorname{det}^{2} \operatorname{Sym}(j)} \in M_{k+l+2, j}\left(\Gamma^{(2)}, \chi \psi\right),
\end{aligned}
$$

by Rankin-Cohen-Ibukiyama type differential operators.

Next we review the differential operator defined by van Dorp [22]. As before, let $k, l$ be positive integers and $\chi, \psi$ characters of $\Gamma^{(2)}$. Let $F \in \bar{M}_{k, j}\left(\Gamma^{(2)}, \chi\right)$ and $g \in M_{l}\left(\Gamma^{(2)}, \psi\right)$ be a vector valued Siegel modular form and a scalar valued Siegel modular form respectively. Then van Dorp 22 constructed

$$
\{F, g\}_{\operatorname{det} \operatorname{Sym}(j)} \in M_{k+l+1, j}\left(\Gamma^{(2)}, \chi \psi\right),
$$

by a differential operator. Though he proved the proposition only when both $\chi$ and $\psi$ are trivial characters, the same proof works for this case.

Finally, we define differential operators on three scalar valued Siegel modular forms. We use these differential operators in order to construct vector valued Siegel modular forms of odd determinant weights from scalar valued Siegel modular forms. For $i=1,2,3$, let $k_{i}$ be a positive integer and $\chi_{i}$ a character of $\Gamma^{(2)}$. For $f_{i} \in$ $M_{k_{i}}\left(\Gamma^{(2)}, \chi_{i}\right)(i=1,2,3)$, we define differential operators as follows:

$$
\begin{aligned}
\left\{f_{1}, f_{2}, f_{3}\right\}_{\operatorname{det} \operatorname{Sym}(j)} & =\left\{\left\{f_{1}, f_{2}\right\}_{\operatorname{Sym}(j)}, f_{3}\right\}_{\operatorname{det} \operatorname{Sym}(j)} \\
& \in M_{k_{1}+k_{2}+k_{3}+1, j}\left(\Gamma^{(2)}, \chi_{1} \chi_{2} \chi_{3}\right), \\
\left\{f_{1}, f_{2}, f_{3}\right\}_{\operatorname{det}^{3} \operatorname{Sym}(j)} & =\left\{\left\{f_{1}, f_{2}\right\}_{\operatorname{det}^{2} \operatorname{Sym}(j)}, f_{3}\right\}_{\operatorname{det} \operatorname{Sym}(j)} \\
& \in M_{k_{1}+k_{2}+k_{3}+3, j}\left(\Gamma^{(2)}, \chi_{1} \chi_{2} \chi_{3}\right),
\end{aligned}
$$

\section{Precise statement of the structure theorems}

In this section, we give generators and relations of $M_{\operatorname{Sym}(10)}^{0}\left(\Gamma^{(2)}\right)$ and $M_{\text {Sym(10) }}^{1}\left(\Gamma^{(2)}\right)$ explicitly. 


\subsection{Generators of $M_{\mathrm{Sym}(10)}^{0}\left(\Gamma^{(2)}\right)$}

In this subsection, we define thirteen generators $M_{\mathrm{Sym}(10)}^{0}\left(\Gamma^{(2)}\right)$. We define ten of them as follows.

$$
\begin{aligned}
F_{10} & =\left\{\varphi_{4}, \varphi_{6}\right\}_{\operatorname{Sym}(10)}, & & F_{12}=\left\{\varphi_{4}, \varphi_{4}^{2}\right\}_{\operatorname{Sym}(10)}, \\
G_{12} & =\left\{\varphi_{4}, \varphi_{6}\right\}_{\operatorname{det}^{2} \operatorname{Sym}(10)}, & F_{14} & =\left\{\varphi_{4}, \varphi_{4} \varphi_{6}\right\}_{\text {Sym }(10)}, \\
G_{14} & =\left\{\varphi_{4}, \chi_{10}\right\}_{\text {Sym }(10)}, & H_{14} & =\left\{\chi_{5}, \varphi_{4} \chi_{5}\right\}_{\text {Sym }(10)}, \\
F_{16} & =\left\{\varphi_{4}, \varphi_{6}^{2}\right\}_{\operatorname{Sym}(10)}, & & G_{16}=\left\{\varphi_{4}, \chi_{12}\right\}_{\text {Sym }(10)}, \\
F_{18} & =\left\{\varphi_{4}, \varphi_{4} \chi_{10}\right\}_{\text {Sym }(10)}, & F_{20} & =\left\{\varphi_{4}, \varphi_{4} \chi_{12}\right\}_{\text {Sym }(10)} .
\end{aligned}
$$

We define remaining three modular forms as follows.

$$
\begin{aligned}
& F_{6}=\frac{1}{667705262220781424640000 \chi_{12}}\left(299617786417098240 F_{18}+1789501343175 B_{18}^{(0)}\right. \\
& -2697884306920857600 B_{18}^{(1)}+4499692750175 B_{18}^{(2)}-3530479328750 B_{18}^{(3)} \\
& +1270685788932672000 B_{18}^{(4)}+310661133307499520 B_{18}^{(5)}+140785854300 B_{18}^{(6)} \\
& +142952698392 B_{18}^{(7)}-153516996000 B_{18}^{(8)}+14151466506240000 B_{18}^{(9)} \\
& \left.-553525841756160000 B_{18}^{(10)}\right) \text {. } \\
& F_{8}=\frac{1}{50077894666555860684800000 \chi_{10}}\left(-8434553345905639680 F_{18}-66731865311475 B_{18}^{(0)}\right. \\
& +16180689061303603200 B_{18}^{(1)}-75624543557850 B_{18}^{(2)}+5670763346875 B_{18}^{(3)} \\
& -14212628597653920000 B_{18}^{(4)}+4104176746346127360 B_{18}^{(5)} \\
& -27222227916975 B_{18}^{(6)}-2824331333544 B_{18}^{(7)}+305313705000 B_{18}^{(8)} \\
& \left.-1559002738648320000 B_{18}^{(9)}-3590159128657920000 B_{18}^{(10)}\right) \text {. } \\
& G_{10}=\frac{1}{13800125293914960000 \varphi_{4}^{2}}\left(-15916405775169019760640 F_{18}-132162848896253175 B_{18}^{(0)}\right. \\
& -36964508172629003366400 B_{18}^{(1)}-61881543692326050 B_{18}^{(2)} \\
& +4575977962943750 B_{18}^{(3)}-20639189761094963520000 B_{18}^{(4)} \\
& +516230561016664865280 B_{18}^{(5)}-23611666345271550 B_{18}^{(6)} \\
& -2865001595829912 B_{18}^{(7)}+215990508690000 B_{18}^{(8)} \\
& -2792613466587732480000 B_{18}^{(9)}-9147741644136775680000 B_{18}^{(10)} \\
& \left.-39157855521483699000000 B_{18}^{(11)}\right) \text {. }
\end{aligned}
$$


Here $B_{18}^{(0)}, \ldots, B_{18}^{(11)}$ are given as follows.

$$
\begin{aligned}
B_{18}^{(0)} & =\left\{\varphi_{4}, \varphi_{4}^{2} \varphi_{6}\right\}_{\operatorname{Sym}(10)}, & B_{18}^{(1)} & =\left\{\chi_{5}, \varphi_{4}^{2} \chi_{5}\right\}_{\operatorname{Sym}(10)}, \\
B_{18}^{(2)} & =\left\{\varphi_{6}, \varphi_{4}^{3}\right\}_{\operatorname{Sym}(10)}, & B_{18}^{(3)} & =\left\{\varphi_{6}, \varphi_{6}^{2}\right\}_{\operatorname{Sym}(10)}, \\
B_{18}^{(4)} & =\left\{\varphi_{6}, \chi_{12}\right\}_{\operatorname{Sym}(10)}, & B_{18}^{(5)} & =\left\{\varphi_{4}^{2}, \chi_{10}\right\}_{\operatorname{Sym}(10)}, \\
B_{18}^{(6)} & =\left\{\varphi_{4}^{2}, \varphi_{4} \varphi_{6}\right\}_{\operatorname{Sym}(10)}, & B_{18}^{(7)} & =\left\{\varphi_{4}, \varphi_{4}^{3}\right\}_{\operatorname{det}^{2} \operatorname{Sym}(10)}, \\
B_{18}^{(8)} & =\left\{\varphi_{4}, \varphi_{6}^{2}\right\}_{\operatorname{det}^{2} \operatorname{Sym}(10)}, & B_{18}^{(9)} & =\left\{\varphi_{4}, \chi_{12}\right\}_{\operatorname{det}^{2} \operatorname{Sym}(10)}, \\
B_{18}^{(10)} & =\left\{\chi_{5}, \varphi_{6} \chi_{5}\right\}_{\operatorname{det}^{2} \operatorname{Sym}(10)}, & B_{18}^{(11)} & =\varphi_{4} \varphi_{6} F_{8} .
\end{aligned}
$$

We note that the set $\left\{F_{18}, B_{18}^{(0)}, \ldots, B_{18}^{(11)}\right\}$ forms a basis of the space $M_{18,10}\left(\Gamma^{(2)}\right)$.

In $\$ 7.3$ we shall explain $F_{6}, F_{8}$ and $F_{10}$ are holomorphic Siegel modular forms.

\subsection{Generators of $M_{\mathrm{Sym}(10)}^{1}\left(\Gamma^{(2)}\right)$}

We define seven of thirteen generators of $M_{\mathrm{Sym}(10)}^{1}\left(\Gamma^{(2)}\right)$ as follows.

$$
\begin{array}{ll}
F_{17}=\left\{\varphi_{4}, \varphi_{6}, \varphi_{6}\right\}_{\operatorname{det} \operatorname{Sym}(10)}, & G_{17}=\left\{\varphi_{4}, \varphi_{4}^{2}, \varphi_{4}\right\}_{\operatorname{det} \operatorname{Sym}(10)}, \\
H_{17}=\left\{\chi_{5}, \varphi_{6}, \chi_{5}\right\}_{\operatorname{det} \operatorname{Sym}(10)}, & F_{19}=\left\{\varphi_{4}, \chi_{5}, \varphi_{4} \chi_{5}\right\}_{\operatorname{det} \operatorname{Sym}(10)}, \\
G_{19}=\left\{\varphi_{4}, \varphi_{4}^{2}, \varphi_{6}\right\}_{\operatorname{det} \operatorname{Sym}(10)}, & F_{21}=\left\{\varphi_{4}, \varphi_{6}, \chi_{10}\right\}_{\operatorname{det} \operatorname{Sym}(10)}, \\
F_{23}=\left\{\varphi_{4}, \varphi_{6}, \chi_{12}\right\}_{\operatorname{det} \operatorname{Sym}(10)} . &
\end{array}
$$

We define remaining six modular forms as follows.

$$
\begin{gathered}
F_{9}=\frac{1}{4636508397286125012516864000 \chi_{10}}\left(-6725180154573619200 F_{19}-7089754342125 G_{19}\right. \\
+22117267502616 B_{19}^{(0)}+4205094643659571200 B_{19}^{(1)}-7138425156720 B_{19}^{(2)} \\
-5140411073357414400 B_{19}^{(3)}-28473519757610188800 B_{19}^{(4)} \\
\left.-78089462464 B_{19}^{(5)}\right) . \\
F_{11}=\frac{1}{670791145440700956672000 \varphi_{4}^{2}}\left(-29326410727288012800 F_{19}-154720948329675 G_{19}\right. \\
-296435594930904 B_{19}^{(0)}+47067973368230707200 B_{19}^{(1)} \\
-357184189630601625600 B_{19}^{(4)}-20661646909696 B_{19}^{(5)} \\
\left.-670791145440700956672000 B_{19}^{(6)}\right) .
\end{gathered}
$$




$$
\begin{aligned}
& F_{13}=\frac{1}{17600933845382823936000 \varphi_{6}}\left(-5870720280512102400 F_{19}+3571610126175 G_{19}\right. \\
& -17576872544088 B_{19}^{(0)}-3898171979838259200 B_{19}^{(1)}+4328987691600 B_{19}^{(2)} \\
& +3418705164194611200 B_{19}^{(3)}+18831546114903244800 B_{19}^{(4)} \\
& \left.-286545870208 B_{19}^{(5)}-17600933845382823936000 B_{19}^{(6)}\right) \text {. } \\
& F_{15}=\frac{1}{13165054156800 \varphi_{4}}\left(258020594380800 F_{19}-12669930 B_{19}^{(2)}+37752728832000 B_{19}^{(3)}\right. \\
& \left.-143718507878400 B_{19}^{(4)}-37349 B_{19}^{(5)}\right) \text {. } \\
& G_{15}=\frac{1}{19508428800 \varphi_{4}} B_{19}^{(0)} . \\
& H_{15}=\frac{1}{92155379097600 \varphi_{4}}\left(594965135278080 B_{19}^{(1)}-944735220 B_{19}^{(2)}+97485935339520 B_{19}^{(3)}\right. \\
& \left.-4267924896614400 B_{19}^{(4)}+550172207 B_{19}^{(5)}\right) .
\end{aligned}
$$

Here $B_{19}^{(0)}, \ldots, B_{19}^{(6)}$ are given by

$$
\begin{array}{ll}
B_{19}^{(0)}=\left\{\varphi_{4}, \varphi_{6}, \varphi_{4}^{2}\right\}_{\operatorname{det} \operatorname{Sym}(10)}, & B_{19}^{(1)}=\left\{\varphi_{4}, \varphi_{4} \chi_{5}, \chi_{5}\right\}_{\operatorname{det} \operatorname{Sym}(10)}, \\
B_{19}^{(2)}=\left\{\varphi_{4}, \varphi_{4} \varphi_{6}, \varphi_{4}\right\}_{\operatorname{det} \operatorname{Sym}(10)}, & B_{19}^{(3)}=\left\{\chi_{5}, \varphi_{4}^{2}, \chi_{5}\right\}_{\operatorname{det} \operatorname{Sym}(10)}, \\
B_{19}^{(4)}=\left\{\chi_{5}, \varphi_{4} \chi_{5}, \varphi_{4}\right\}_{\operatorname{det} \operatorname{Sym}(10)}, & B_{19}^{(5)}=\left\{\varphi_{6}, \varphi_{4}^{2}, \varphi_{4}\right\}_{\operatorname{det} \operatorname{Sym}(10)}, \\
B_{19}^{(6)}=\varphi_{4} \varphi_{6} F_{9} . &
\end{array}
$$

We note that the set $\left\{F_{19}, G_{19}, B_{19}^{(0)}, \ldots, B_{19}^{(6)}\right\}$ forms a basis of the space $M_{19,10}\left(\Gamma^{(2)}\right)$. In $\$ 7.3$, we shall explain that $F_{9}, F_{11}, F_{13}, F_{15}, G_{15}$ and $H_{15}$ are holomorphic modular forms.

\subsection{Relations of $M_{\mathrm{Sym}(10)}^{0}\left(\Gamma^{(2)}\right)$}

Lemma 6.1. Generators of even determinant weights satisfy the following two relations.

$$
\begin{aligned}
& a_{18} F_{6}+a_{16} F_{8}+a_{14} F_{10}+b_{14} G_{10}+a_{12} F_{12}+b_{12} G_{12}+a_{10} F_{14}+b_{10} G_{14} \\
& +c_{10} H_{14}+a_{8} F_{16}+b_{8} G_{16}+a_{6} F_{18}+a_{4} F_{20}=0
\end{aligned}
$$

and

$$
\begin{aligned}
& \alpha_{20} F_{6}+\alpha_{18} F_{8}+\alpha_{16} F_{10}+\beta_{16} G_{10}+\alpha_{14} F_{12}+\beta_{14} G_{12}+\alpha_{12} F_{14}+\beta_{12} G_{14} \\
& +\gamma_{12} H_{14}+\alpha_{10} F_{16}+\beta_{10} G_{16}+\alpha_{8} F_{18}+\alpha_{6} F_{20}=0 .
\end{aligned}
$$

Here $a_{18}$ and $a_{16}$ are defined by

$a_{18}=15643660032\left(67331642279 \varphi_{4}^{3} \varphi_{6}-6689228000 \varphi_{6}^{3}+2370923189526528 \varphi_{4}^{2} \chi_{10}+4612035407616000 \varphi_{6} \chi_{12}\right)$, 
and

$a_{16}=5105916816000\left(387254979 \varphi_{4}^{4}-20240608 \varphi_{4} \varphi_{6}^{2}+6135186530304 \varphi_{6} \chi_{10}+4114923337728 \varphi_{4} \chi_{12}\right)$.

Coefficients $a_{14}, \ldots, a_{4}$ are defined by

$$
\begin{gathered}
a_{14}=-11824384 \varphi_{4}\left(-392419241 \varphi_{4} \varphi_{6}+145847127883968 \chi_{10}\right), \\
b_{14}=-1986496512 \varphi_{4}\left(-31914599 \varphi_{4} \varphi_{6}+10054388155392 \chi_{10}\right), \\
a_{12}=830289075\left(23924401 \varphi_{4}^{3}-1654848 \varphi_{6}^{2}+1331133253632 \chi_{12}\right), \\
b_{12}=76257199200\left(23313 \varphi_{4}^{3}-2080 \varphi_{6}^{2}+1434101760 \chi_{12}\right), \\
a_{10}=-135273640320\left(5849 \varphi_{4} \varphi_{6}+412304256 \chi_{10}\right), \\
b_{10}=41009272012800\left(-909943 \varphi_{4} \varphi_{6}+346335575040 \chi_{10}\right), \\
c_{10}=261554147777367244800\left(-\varphi_{4} \varphi_{6}+3468906 \chi_{10}\right), \\
a_{8}=312420856840400 \varphi_{4}^{2}, \\
b_{8}=70552675506957557760 \varphi_{4}^{2}, \\
a_{6}=-89694864607315968000 \varphi_{6}, \\
a_{4}=165176542976857251840 \varphi_{4} .
\end{gathered}
$$

Coefficients $\alpha_{20}, \alpha_{18}, \alpha_{16}$ and $\beta_{16}$ are defined by $\alpha_{20}=-5214553344\left(425048543296 \phi_{4}^{5}+484587670889 \phi_{4}^{2} \phi_{6}^{2}+15696098336318400 \phi_{4} \phi_{6} \chi_{10}\right.$ $\left.+62924853322985472 \phi_{4}^{2} \chi_{12}-890719685310873600000 \chi_{10}^{2}\right)$,

$\alpha_{18}=5105916816000\left(-1811050991 \phi_{4}^{3} \phi_{6}-24020864 \phi_{6}^{3}-145283115931200 \phi_{4}^{2} \chi_{10}+87510477938688 \phi_{6} \chi_{12}\right)$, $\alpha_{16}=5912192\left(19495410909 \phi_{4}^{4}-23419603319 \phi_{4} \phi_{6}^{2}-1950560293550400 \phi_{6} \chi_{10}+2818879454297088 \phi_{4} \chi_{12}\right)$, and

$\beta_{16}=1986496512\left(649847848 \phi_{4}^{4}-809420843 \phi_{4} \phi_{6}^{2}-86364658900800 \phi_{6} \chi_{10}+87573208859136 \phi_{4} \chi_{12}\right)$.

Coefficients $\alpha_{14}, \ldots, \alpha_{6}$ are defined by

$$
\begin{gathered}
\alpha_{14}=-4151445375 \phi_{4}\left(22269553 \phi_{4} \phi_{6}+1622175242688 \chi_{10}\right), \\
\beta_{14}=-381285996000 \phi_{4}\left(21233 \phi_{4} \phi_{6}+1927074240 \chi_{10}\right), \\
\alpha_{12}=-135273640320\left(14911 \phi_{4}^{3}-44156 \phi_{6}^{2}+3872074752 \chi_{12}\right), \\
\beta_{12}=-205046360064000\left(2855021 \phi_{4}^{3}-3764964 \phi_{6}^{2}+718771802112 \chi_{12}\right), \\
\gamma_{12}=-16347134236085452800\left(1649 \phi_{4}^{3}-1729 \phi_{6}^{2}+211323168 \chi_{12}\right), \\
\alpha_{10}=11238160318000\left(-139 \phi_{4} \phi_{6}+11793600 \chi_{10}\right), \\
\beta_{10}=-59317870781030400\left(5947 \phi_{4} \phi_{6}+1014249600 \chi_{10}\right), \\
\alpha_{8}=448474323036579840000 \phi_{4}^{2}, \\
\alpha_{6}=-825882714884286259200 \phi_{6} .
\end{gathered}
$$

We shall prove Lemma 6.1 in \$7.4 


\subsection{Relations of $M_{\mathrm{Sym}(10)}^{1}\left(\Gamma^{(2)}\right)$}

Lemma 6.2. Generators of odd determinant weights satisfy the following two relations.

$$
\begin{aligned}
& d_{18} F_{9}+d_{16} F_{11}+d_{14} F_{13}+d_{12} F_{15}+e_{12} G_{15}+f_{12} H_{15}+d_{10} F_{17}+e_{10} G_{17} \\
& \quad+f_{10} H_{17}+d_{8} F_{19}+e_{8} G_{19}+d_{6} F_{21}+d_{4} F_{23}=0
\end{aligned}
$$

and

$$
\begin{aligned}
& \delta_{20} F_{9}+\delta_{18} F_{11}+\delta_{16} F_{13}+\delta_{14} F_{15}+\epsilon_{14} G_{15}+\zeta_{14} H_{15}+\delta_{12} F_{17}+\epsilon_{12} G_{17} \\
& \left.\quad+\zeta_{12} H_{17}+\delta_{10} F_{19}+\epsilon_{10} G_{19}+\delta_{8} F_{21}+\delta_{6} F_{23}=0.4\right)
\end{aligned}
$$

Here $d_{18}$ and $d_{16}$ are defined by

$d_{18}=-195284202946560\left(-199029244391 \phi_{4}^{3} \phi_{6}+2697950850992640 \phi_{4}^{2} \chi_{10}+3549133607846400 \phi_{6} \chi_{12}\right)$, and

$d_{16}=-341747355156480\left(6216500122 \phi_{4}^{4}-25056024021 \phi_{4} \phi_{6}^{2}-471257790566400 \phi_{6} \chi_{10}\right.$

$$
\left.+2428645469322240 \phi_{4} \chi_{12}\right) \text {. }
$$

Coefficients $d_{14}, \ldots, d_{4}$ are defined by

$$
\begin{gathered}
d_{14}=-74893556325778260295680 \phi_{4}\left(-433 \phi_{4} \phi_{6}+14131200 \chi_{10}\right) \\
d_{12}=-102195265536\left(-685230911 \phi_{4}^{3}+35309376921600 \chi_{12}\right) \\
e_{12}=-1093591536500736\left(39827745779 \phi_{4}^{3}+468263910297600 \chi_{12}\right), \\
f_{12}=-255488163840\left(193718497 \phi_{4}^{3}+4258403020800 \chi_{12}\right) \\
d_{10}=955493351615296\left(-49 \phi_{4} \phi_{6}+3686400 \chi_{10}\right) \\
e_{10}=387290963292597\left(7 \phi_{4} \phi_{6}+115200 \chi_{10}\right) \\
f_{10}=5177826116350101946368\left(-7 \phi_{4} \phi_{6}+460800 \chi_{10}\right) \\
d_{8}=-2133719553440976076800 \phi_{4}^{2} \\
e_{8}=0 \\
d_{6}=-396262202781895557120 \phi_{6} \\
d_{4}=-66043700463649259520 \phi_{4}
\end{gathered}
$$

Coefficients $\delta_{20}, \delta_{18}$ and $\delta_{16}$ are defined by

$\delta_{20}=-27897743278080\left(-1439954360064 \phi_{4}^{5}+46749649327 \phi_{4}^{2} \phi_{6}^{2}+30085945775101440 \phi_{4} \phi_{6} \chi_{10}\right.$

$$
\left.+34225097039884800 \phi_{4}^{2} \chi_{12}-7541542192496423731200 \chi_{10}^{2}\right),
$$

$$
\begin{array}{r}
\delta_{18}=341747355156480\left(16282786754 \phi_{4}^{3} \phi_{6}+2556737145 \phi_{6}^{3}-5088306946498560 \phi_{4}^{2} \chi_{10}\right. \\
\left.+895389802076160 \phi_{6} \chi_{12}\right),
\end{array}
$$

and

$\delta_{16}=-10699079475111180042240\left(-3036 \phi_{4}^{4}+5 \phi_{4} \phi_{6}^{2}-49459200 \phi_{6} \chi_{10}+130636800 \phi_{4} \chi_{12}\right)$. 
Coefficients $\delta_{14}, \ldots, \delta_{6}$ are defined by

$$
\begin{gathered}
\delta_{14}=-8073425977344 \phi_{4}\left(-8673809 \phi_{4} \phi_{6}+260218327680 \chi_{10}\right), \\
\epsilon_{14}=-1093591536500736 \phi_{4}\left(39827745779 \phi_{4} \phi_{6}+659445588817920 \chi_{10}\right), \\
\zeta_{14}=-255488163840 \phi_{4}\left(193718497 \phi_{4} \phi_{6}+11030709116160 \chi_{10}\right), \\
\delta_{12}=955493351615296\left(-44 \phi_{4}^{3}-5 \phi_{6}^{2}+4838400 \chi_{12}\right), \\
\epsilon_{12}=-55327280470371\left(-44 \phi_{4}^{3}-5 \phi_{6}^{2}+967680 \chi_{12}\right), \\
\zeta_{12}=739689445192871706624\left(-44 \phi_{4}^{3}-5 \phi_{6}^{2}+4193280 \chi_{12}\right), \\
\delta_{10}=2133719553440976076800\left(-\phi_{4} \phi_{6}+64512 \chi_{10}\right), \\
\epsilon_{10}=-35692735177045739520 \chi_{10}, \\
\delta_{8}=-396262202781895557120 \phi_{4}^{2}, \\
\delta_{6}=-66043700463649259520 \phi_{6} .
\end{gathered}
$$

We shall prove Lemma 6.2 in $\$ 7.4$.

\section{Proof of the main results}

In this section, we prove Theorem 1.1 and Theorem 1.2. Also we prove generators given in \$6 are holomorphic modular forms. The actual computation is done by a computer algebra system. We use SageMath [18, and a SageMath package for Siegel modular forms degree two $[19$.

\subsection{Hilbert series}

For $j \in \mathbb{Z}_{\geq 0}$ and $\epsilon \in\{0,1\}$, we define the Hilbert series $h_{j, \epsilon}(t)$ of $M_{\operatorname{Sym}(j)}\left(\Gamma^{(2)}\right)$ by

$$
h_{j, \epsilon}(t)=\sum_{k=0}^{\infty} \operatorname{dim}_{\mathbb{C}} M_{k, j}\left(\Gamma^{(2)}\right) t^{k} .
$$

Then $h_{10, \epsilon}(t)$ is given as follows.

\section{Lemma 7.1 .}

$$
\begin{aligned}
& h_{10,0}(t)=\frac{t^{6}+t^{8}+2 t^{10}+2 t^{12}+3 t^{14}+2 t^{16}+t^{18}+t^{20}-t^{24}-t^{26}}{\left(1-t^{4}\right)\left(1-t^{6}\right)\left(1-t^{10}\right)\left(1-t^{12}\right)}, \\
& h_{10,1}(t)=\frac{t^{9}+t^{11}+t^{13}+3 t^{15}+3 t^{17}+2 t^{19}+t^{21}+t^{23}-t^{27}-t^{29}}{\left(1-t^{4}\right)\left(1-t^{6}\right)\left(1-t^{10}\right)\left(1-t^{12}\right)} .
\end{aligned}
$$

In particular, expansions of these Hilbert series are given by

$$
\begin{aligned}
h_{10,0}(t)=t^{6}+t^{8}+3 t^{10}+4 t^{12} & +7 t^{14}+9 t^{16}+13 t^{18} \\
& +17 t^{20}+22 t^{22}+27 t^{24}+35 t^{26}+41 t^{28}+O\left(t^{30}\right)
\end{aligned}
$$

and

$$
\begin{aligned}
h_{10,1}(t)=t^{9}+t^{11}+2 t^{13}+5 t^{15} & +6 t^{17}+9 t^{19} \\
+ & 13 t^{21}+16 t^{23}+21 t^{25}+28 t^{27}+33 t^{29}+O\left(t^{31}\right) .
\end{aligned}
$$


Proof. $h_{10,0}(t)$ is given in [7]. But for the sake of completeness, we give a proof. By Tsushima 21], the dimension of $S_{k, j}\left(\Gamma^{(2)}\right)$ is known if $k>4$. By Arakawa [2], we can compute $\operatorname{dim}_{\mathbb{C}} M_{k, j}\left(\Gamma^{(2)}\right)-\operatorname{dim}_{\mathbb{C}} S_{k, j}\left(\Gamma^{(2)}\right)$ if $k>4$. Therefore we have to prove $M_{k, 10}\left(\Gamma^{(2)}\right)=0$ if $k \leq 4$. The vanishing of $M_{k, 10}\left(\Gamma^{(2)}\right)$ for $k \leq 0$ follows from Freitag's work 4]. We prove $M_{k, 10}\left(\Gamma^{(2)}\right)=S_{k, 10}\left(\Gamma^{(2)}\right)$ for $1 \leq k \leq 4$. If $k$ is odd, this follows from the definition. By Ibukiyama [7], $M_{2,10}\left(\Gamma^{(2)}\right)=S_{2,10}\left(\Gamma^{(2)}\right)$. Since $S_{14}\left(\mathrm{SL}_{2}(\mathbb{Z})\right)=0$, we have $M_{4,10}\left(\Gamma^{(2)}\right)=S_{4,10}\left(\Gamma^{(2)}\right)$. Thus we have $M_{k, 10}\left(\Gamma^{(2)}\right)=$ $S_{k, 10}\left(\Gamma^{(2)}\right)$ for $1 \leq k \leq 4$. Again, by Ibukiyama 7 , we have $S_{k, 10}\left(\Gamma^{(2)}\right)=0$ if $0 \leq k \leq 4$. Therefore we have $M_{k, 10}\left(\Gamma^{(2)}\right)=0$ if $0 \leq k \leq 4$.

\subsection{Precision}

We explain to what extent we compute Fourier coefficients of the generators. For $f \in M_{k}\left(\Gamma^{(2)}\right)$, we consider $f$ as an element of $\left.\mathbb{C}\left[q_{12}, q_{12}^{-1}\right] \llbracket q_{11}, q_{22}\right]$ as in $\$ 2$

We recall Sturm type theorem for scalar valued Siegel modular forms of degree 2 for our need. We introduce some notation. For $f \in \mathbb{C}\left[q_{12}, q_{12}^{-1}\right] \llbracket q_{11}, q_{22} \rrbracket$, we denote coefficients of $f$ as follows:

$$
f=\sum_{(n, r, m)} a((n, r, m) ; f) q_{11}^{n} q_{12}^{r} q_{22}^{m} .
$$

For $a \in \mathbb{R}_{\geq 0}$ and a subring $B \subset \mathbb{C}$, we define a ring by

$$
R_{a}(B)=R^{\prime}(B) /\left(q_{11}^{[a]+1}, q_{22}^{[a]+1}\right) .
$$

Here [·] is the Gauss symbol and $R^{\prime}(B)$ is defined by

$$
R^{\prime}(B)=\left\{f \in B\left[q_{12}, q_{12}^{-1}\right] \llbracket q_{11}, q_{22} \rrbracket \mid a((n, r, m) ; f)=0 \text { if } 4 n m-r^{2}<0\right\} .
$$

Theorem 7.1 (Kikuta-Takemori $[12]$ ). Let $k \in \mathbb{Z}_{\geq 0}$ and $p$ a prime number. Suppose $f \in M_{k}\left(\Gamma^{(2)}\right)$ has $\mathbb{Z}_{(p)}$ integral Fourier coefficients. We put

$$
\left.\bar{f}=\text { The image of } f \text { in } \mathbb{Z} / p \mathbb{Z}\left[q_{12}, q_{12}^{-1}\right] \llbracket q_{11}, q_{22}\right]
$$

and

$$
b_{k}= \begin{cases}k / 10 & \text { if } k \text { is even, } \\ (k-5) / 10 & \text { if } k \text { is odd. }\end{cases}
$$

If the image of $\bar{f}$ in $R_{b_{k}}(\mathbb{Z} / p \mathbb{Z})$ vanishes, then we have $\bar{f}=0$.

By the theorem above and the fact that $M_{k}\left(\Gamma^{(2)}\right)$ has a basis of modular forms with integral Fourier coefficients, we have the following lemma.

Lemma 7.2. Let $f \in M_{k}\left(\Gamma^{(2)}\right)$ and assume the image of $f$ in $R_{b_{k}}(\mathbb{C})$ vanishes, where $b_{k}$ is as in Theorem 7.1. Then we have $f=0$.

Remark 7.1. The statement of $[20]$ is true, but the proof is not sufficient. 
In Lemma 7.5, we shall prove the determinant of the first 11 generators (ordered by the determinant weight) of $M_{\mathrm{Sym}(10)}^{0}\left(\Gamma^{(2)}\right)\left(\right.$ resp. $\left.M_{\mathrm{Sym}(10)}^{1}\left(\Gamma^{(2)}\right)\right)$ is equal to $\chi_{35}^{5}\left(\phi_{4}^{3}-\phi_{6}^{2}\right)\left(\right.$ resp. $\left.\chi_{35}^{6}\left(\phi_{4}^{3}-\phi_{6}^{2}\right)\right)$ up to a non-zero constant. By Lemma 7.2 it is enough to compute generators of $M_{\operatorname{Sym}(10)}^{0}\left(\Gamma^{(2)}\right)\left(\right.$ resp. $\left.M_{\operatorname{Sym}(10)}^{1}\left(\Gamma^{(2)}\right)\right)$ in $R_{18}(\mathbb{C}) \otimes_{\mathbb{C}} V_{10}\left(\right.$ resp. $\left.R_{22}(\mathbb{C}) \otimes_{\mathbb{C}} V_{10}\right)$.

\subsection{Holomorphy of generators}

In $\$ 6$ we constructed generators of small determinant weights by dividing modular forms. Since dividing by a cusp form is subtle, we introduce the following lemma.

Lemma 7.3. For $a \in \mathbb{Z}_{\geq 1}$, let $R_{a}(\mathbb{C})$ be the ring defined by (7.1). Let

$$
\begin{aligned}
& f=\sum_{(n, r, m)} a((n, r, m) ; f) q_{11}^{n} q_{12}^{r} q_{22}^{m} \bmod \left(q_{11}^{a+1}, q_{22}^{a+1}\right), \\
& g=\sum_{(n, r, m)} a((n, r, m) ; g) q_{11}^{n} q_{12}^{r} q_{22}^{m} \bmod \left(q_{11}^{a+1}, q_{22}^{a+1}\right),
\end{aligned}
$$

be elements of $R_{a}(\mathbb{C})$. Assume $a((1,-1,1) ; g)=1$ and $g$ is cuspidal, that is $a((n, r, m) ; g)=0$ if $4 n m-r^{2}=0$ and $n, m \leq a$. Then there uniquely exists $h \in R_{a-1}(\mathbb{C})$ such that $\widetilde{f}=\widetilde{g} h$. Here $\widetilde{f}$ (resp. $\widetilde{g}$ ) is the image of $f$ (resp. $g$ ) in $R_{a-1}(\mathbb{C})$. Moreover, we have the following recursive equation.

$$
\begin{aligned}
& \quad a((n, r, m) ; h)+a((1,1,1) ; g) a((n, r-2, m) ; h) \\
& \qquad=a((n+1, r-1, m+1) ; f), \\
& \text { for }(n, r, m) \in \mathbb{Z}^{3} \text { with } 0 \leq n, m \leq a-1 \text { and } 4 n m-r^{2} \geq 0 .
\end{aligned}
$$

Proof. We put $S=\left\{(n, r, m) \in \mathbb{Z}^{3} \mid n, m, 4 n m-r^{2} \geq 0\right\}$. We define an order $\leq$ of $S$ so that $(n, r, m) \leq\left(n^{\prime}, r^{\prime}, m^{\prime}\right)$ if and only if one of following conditions holds.

(1) $n+m \leq n^{\prime}+m^{\prime}$.

(2) $n+m=n^{\prime}+m^{\prime}$ and $n \leq n^{\prime}$.

(3) $n=n^{\prime}, m=m^{\prime}$ and $r \leq r^{\prime}$.

Let $h=\sum_{(n, r, m) \geq 0} a((n, r, m) ; h) q_{11}^{n} q_{12}^{r} q_{22}^{m}+\bmod \left(q_{11}^{a}, q_{22}^{a}\right)$ be an element of $R_{a-1}(\mathbb{C})$. By the condition $\tilde{f}=\widetilde{g} h$, we have

$$
\sum_{\substack{T_{1}+T_{2}=(n+1, r-1, m+1) \\ T_{1}, T_{2} \in S}} a\left(T_{1} ; g\right) a\left(T_{2} ; h\right)=a((n+1, r-1, m+1) ; f),
$$

for $(n, r, m) \in S$ with $n, m \leq a-1$. Here addition of $S$ is defined by entry-wise. By (7.3) and the cuspidality of $g$, we obtain 7.2 . Thus we can recursively define $a((n, r, m) ; h)$ for $(n, r, m) \in S$ with $n, m \leq a-1$ by the order of $S$. Uniqueness follows from the recursive equation above.

Next, we prove holomorphy of generators. 
Lemma 7.4. Meromorphic modular forms $F_{6}, F_{8}, G_{10}, F_{9}, F_{11}, F_{13}, F_{15}$ and $H_{15}$ given in \$6.1 and \$6.2 are holomorphic.

Proof. For simplicity, we only prove $F_{9}$ is holomorphic. We can prove other cases in a similar way. Let $N_{19}$ be the subspace of $M_{19,10}\left(\Gamma^{(2)}\right)$ spanned by $F_{19}, G_{19}, B_{19}^{(0)}, \ldots, B_{19}^{(5)}$. Then $N_{19}$ is a subspace of $M_{19,10}\left(\Gamma^{(2)}\right)$ of codimension 1. And we can calculate a basis of $N_{19}$ explicitly from Fourier coefficients of scalar valued Siegel modular forms. Put $C_{19}=F_{19} \mid T(2)$, where $T(2)$ is the Hecke operator. For the explicit action of $T(2)$ on $M_{k, j}\left(\Gamma^{(2)}\right)$, see [2]. Then we have $N_{19}+\mathbb{C} C_{19}=M_{19,10}\left(\Gamma^{(2)}\right)$. We can check this equality by computing vector valued modular forms in $R_{6}(\mathbb{Q})^{11}$. By Lemma 7.1 there uniquely exists $F_{9}^{\prime} \in M_{9,10}\left(\Gamma^{(2)}\right)$ such that $F_{9}^{\prime} \neq 0$ up to a non-zero constant. By a similar argument in $[1]$ we can explicitly find rational numbers $\alpha, \beta, \gamma, \delta, c^{(i)}, d^{(i)}$ for $-1 \leq i \leq 5$ such that

$$
\begin{aligned}
\phi_{4} \phi_{6} F_{9}^{\prime} & =\alpha F_{19}+\beta C_{19}+c^{(-1)} G_{19}+\sum_{i=0}^{5} c^{(i)} B_{19}^{(i)}, \\
\phi_{10} F_{9}^{\prime} & =\gamma F_{19}+\delta C_{19}+d^{(-1)} G_{19}+\sum_{i=0}^{5} d^{(i)} B_{19}^{(i)} .
\end{aligned}
$$

Then we have $\beta=\delta \neq 0$ and $\alpha \neq \gamma$. Since $C_{19}$ is computationally expensive, we remove $C_{19}$. Then we have

$$
\chi_{10} F_{9}^{\prime}=\alpha^{\prime} F_{19}+\beta^{\prime} G_{19}+\sum_{i=0}^{5} e^{(i)} B_{19}^{(i)},
$$

for some rational numbers $\alpha^{\prime}, \beta^{\prime}, e^{(i)}(0 \leq i \leq 5)$. By explicit computation of these rational numbers, we have $F_{9}^{\prime}=F_{9}$ up to a non-zero constant. Thus $F_{9}$ is holomorphic.

\subsection{Relations}

In this subsection, we prove Lemma 6.1 and Lemma 6.2 For simplicity, we only prove relation 6.1.

Proof. [Proof of relation 6.1] ] For $k \in \mathbb{Z}_{\geq 0}$, let $X_{k}$ be the following set of monomial of $\phi_{4}, \phi_{6}, \chi_{10}$ and $\chi_{12}$;

$$
X_{k}=\left\{\phi_{4}^{a} \phi_{6}^{b} \chi_{10}^{c} \chi_{12}^{d} \mid a, b, c, d \in \mathbb{Z}_{\geq 0}, 4 a+6 b+10 c+12 d=k\right\} .
$$

We define a finite set $S$ by

$$
\begin{aligned}
& X_{18} F_{6} \cup X_{16} F_{8} \cup X_{14} F_{10} \cup X_{14} G_{10} \\
& \qquad X_{12} F_{12} \cup X_{12} G_{12} \cup X_{10} F_{14} \cup X_{10} G_{14} \\
& \cup X_{10} H_{14} \cup X_{8} F_{16} \cup X_{8} G_{16} \cup X_{6} F_{18} .
\end{aligned}
$$


Then we have $|S|=27$. By computing elements of $S$ in $R_{6}(\mathbb{C}) \otimes_{\mathbb{C}} V_{10}$, we see that $S$ is linearly independent over $\mathbb{C}$. By Lemma 7.1 $S$ is a basis of $M_{24,10}\left(\Gamma^{(2)}\right)$. By numerical computation, we can check that the image of the left hand side of 6.1) in $R_{6}(\mathbb{C}) \otimes_{\mathbb{C}} V_{10}$ is equal to 0 . Therefore the left hand side of $(6.1)$ is equal to 0 .

\subsection{Determinants of generators and structure theorems}

Determinants of modular forms given in $\$ 6.1$ and 6.2 are given as follows.

\section{Lemma 7.5.}

$$
F_{6} \wedge F_{8} \wedge F_{10} \wedge G_{10} \wedge F_{12} \wedge G_{12} \wedge F_{14} \wedge G_{14} \wedge H_{14} \wedge F_{16} \wedge G_{16}=\left(\phi_{4}^{3}-\phi_{6}^{2}\right) \chi_{35}^{5},
$$

up to a non-zero constant and

$$
F_{9} \wedge F_{11} \wedge F_{13} \wedge F_{15} \wedge G_{15} \wedge H_{15} \wedge F_{17} \wedge G_{17} \wedge H_{17} \wedge F_{19} \wedge G_{19}=\left(\phi_{4}^{3}-\phi_{6}^{2}\right) \chi_{35}^{6},
$$

up to a non-zero constant.

Proof. As is explained in $\$ 7.2$ we have to compute both sides of 7.4 (resp. $(7.5)$ ) in $R_{18}(\mathbb{Q})$ (resp. $R_{22}(\mathbb{Q})$ ). Computation is done by using SageMath $[18]$ and a package [19] for Siegel modular forms of degree 2. See https://github.com/ stakemori/det_vec_vald_SMFs for a script to check this.

Next we prove Theorem 1.1

Theorem 7.2. Theorem 1.1 is true, that is the sequences (1.1) and 1.2 are exact. Here the second linear map of the sequence (1.1) is given by sending the standard basis to $\left(a_{18}, a_{16}, \ldots, a_{4}\right)$ and $\left(\alpha_{20}, \alpha_{18}, \ldots, \alpha_{6}\right)$, where $a_{18}, a_{16}, \ldots, a_{4} ; \alpha_{20}, \alpha_{18}, \ldots, \alpha_{6}$ are give in (6.1) and 6.2). The third map of the sequence (1.1) is given by sending the standard basis to $F_{6}, F_{8}, \ldots, F_{20}$. Linear maps of the sequence 1.2 is given in a similar way by using $F_{9}, F_{11}, \ldots, H_{15}$, 6.3 and 6.4.

Proof. For simplicity, we only prove that 1.1 is exact. We denote by $\psi_{-2}$ the second map of 1.1 and by $\psi_{-1}$ the third map of 1.1. Because

$$
\operatorname{det}\left(\begin{array}{cc}
a_{6} & a_{4} \\
\alpha_{8} & \alpha_{6}
\end{array}\right)=\phi_{4}^{3}-\phi_{6}^{2}
$$

up to a non-zero constant, $\psi_{-2}$ is injective. By Lemma 6.1, we have $\operatorname{Im}\left(\psi_{-2}\right) \subset$ $\operatorname{ker}\left(\psi_{-1}\right)$. By 1.3 and Lemma 7.5 .

$$
\left\{F_{6}, F_{8}, F_{10}, G_{10}, F_{12}, G_{12}, F_{14}, G_{14}, H_{14}, F_{16}, G_{16}\right\}
$$

is a basis of $M_{\mathrm{Sym}(10)}^{0}\left(\Gamma^{(2)}\right) \otimes_{A_{\mathrm{ev}}} K_{\mathrm{ev}}$. Therefore we have

$$
\left(\operatorname{ker}\left(\psi_{-1}\right) / \operatorname{Im}\left(\psi_{-2}\right)\right) \otimes_{A_{\mathrm{ev}}} K_{\mathrm{ev}}=\{0\} .
$$


Since $\operatorname{det}\left(\begin{array}{ll}a_{6} & a_{4} \\ \alpha_{8} & \alpha_{6}\end{array}\right)$ and $\operatorname{det}\left(\begin{array}{cc}b_{8} & a_{4} \\ \beta_{10} & \alpha_{6}\end{array}\right)$ are co-prime in $A_{\mathrm{ev}}$, we have $\operatorname{ker}\left(\psi_{-1}\right)=$ $\operatorname{Im}\left(\psi_{-2}\right)$. Therefore the Hilbert series of $\operatorname{Im}\left(\psi_{-1}\right)$ is equal to

$$
\frac{t^{6}+t^{8}+2 t^{10}+2 t^{12}+3 t^{14}+2 t^{16}+t^{18}+t^{20}-t^{24}-t^{26}}{\left(1-t^{4}\right)\left(1-t^{6}\right)\left(1-t^{10}\right)\left(1-t^{12}\right)},
$$

which is equal to that of $M_{\mathrm{Sym}(10)}^{0}\left(\Gamma^{(2)}\right)$ by Lemma 7.1 . Therefore $\psi_{-1}$ is surjective. This completes the proof.

Finally, we prove Theorem 1.2

Proof. [Proof of Theorem 1.2] By Proposition 4.3, it is enough to prove the assertion when $j=10$. We can prove the assertion of the theorem by a similar argument to the proof of Proposition 4.3 by using Theorem 4.1. Lemma 7.5 and 7.6 .

\section{Examples and a table of Fourier coefficients}

Since constructions of modular forms given in $\$ 6.1$ and 6.2 are complicated, the author has tested them in several ways. By the explicit structure theorems, we can construct a basis of the space $M_{k, 10}\left(\Gamma^{(2)}\right)$ and compute the action of Hecke operators on the space. In the case when Hecke eigenvalues are known, we test if eigenforms have correct eigenvalues. We also test the generalized Ramanujan conjecture for a non CAP cuspidal eigenform.

For tests, we introduce the following notation. Let $F \in M_{k, j}\left(\Gamma^{(2)}\right)$ be a Hecke eigenform and $p$ a prime number. We denote by $Q_{p}(F ; X)$ the polynomial of degree 4 of $X$ so that $\prod_{p}$ :prime $Q_{p}\left(F ; p^{-s}\right)^{-1}$ is the spinor $L$-function of $F$ and denote by $R_{p}(F ; X)$ the polynomial of degree 5 of $X$ so that $\prod_{p: \text { prime }} R_{p}\left(F ; p^{-s}\right)^{-1}$ is the standard $L$-function of $F$. That is, $Q_{p}(F ; X)$ and $R_{p}(F ; X)$ are defined as follows.

$$
\begin{gathered}
Q_{p}(F ; X)=\left(1-\alpha_{0} X\right)\left(1-\alpha_{0} \alpha_{1} X\right)\left(1-\alpha_{0} \alpha_{2} X\right)\left(1-\alpha_{0} \alpha_{1} \alpha_{2} X\right), \\
R_{p}(F ; X)=(1-X)\left(1-\alpha_{1} X\right)\left(1-\alpha_{1}^{-1} X\right)\left(1-\alpha_{2} X\right)\left(1-\alpha_{2}^{-1} X\right) .
\end{gathered}
$$

Here $\alpha_{0}, \alpha_{1}, \alpha_{2}$ are the $p$-Satake parameters of $F$.

\subsection{Test for Klingen-Eisenstein series and the $K-R-S$ lift}

Since Hecke eigenvalues of Eisenstein series and lifts are known, we test them. Let $k, j \in 2 \mathbb{Z}_{\geq 0}$. For a normalized eigenform $f=\sum_{n=1}^{\infty} a(n ; f) q^{n} \in S_{k+j}\left(\mathrm{SL}_{2}(\mathbb{Z})\right)$ with $k>4$, Arakawa [2] defined the Klingen-Eisenstein series $E(f) \in M_{k, j}\left(\Gamma^{(2)}\right)$. The Klingen-Eisenstein series $E(f)$ satisfies the following identities:

$$
\begin{gathered}
\Phi(E(f))=f u_{1}^{j}, \\
Q_{p}(E(f) ; X)=Q_{p}^{(1)}(f ; X) Q_{p}^{(1)}\left(f ; p^{(k-2)} X\right) .
\end{gathered}
$$

Here $p$ is a prime number and $Q_{p}^{(1)}(f ; X)=1-a(p ; f) X+p^{k+j-1} X^{2}$. 
For a normalized eigenform $f=\sum_{n=1}^{\infty} a(n ; f) q^{n} \in S_{k}\left(\mathrm{SL}_{2}(\mathbb{Z})\right)$, Ramakrishnan and Shahidi 16 constructed a lift $\mathrm{KS}(f) \in M_{k+1, k-2}\left(\mathrm{SL}_{2}(\mathbb{Z})\right)$ called the KimRamakrishnan-Shahidi lift (K-R-S lift in short). The K-R-S lift KS $(f)$ satisfies the following identity:

$$
Q_{p}(\mathrm{KS}(f) ; X)=\prod_{i=0}^{3}\left(1-\alpha_{p}^{i} \beta_{p}^{3-i} X\right)
$$

where $p$ is a prime number. $\alpha_{p}$ and $\beta_{p}$ are defined by $1-a(p ; f) X+p^{k-1} X^{2}=$ $\left(1-\alpha_{p} X\right)\left(1-\beta_{p} X\right)$.

Let

$$
\begin{gathered}
\Delta=q-24 q^{2}+252 q^{3}-1472 q^{4}+4830 q^{5}+O\left(q^{6}\right) \in S_{12}\left(\mathrm{SL}_{2}(\mathbb{Z})\right), \\
f_{16}=q+216 q^{2}-3348 q^{3}+13888 q^{4}+52110 q^{5}+O\left(q^{6}\right) \in S_{16}\left(\mathrm{SL}_{2}(\mathbb{Z})\right)
\end{gathered}
$$

be normalized eigenforms of weight 12 and 16 respectively. Then we have

$$
F_{6}=E\left(f_{16}\right), \quad F_{13}=\mathrm{KS}(\Delta) .
$$

Fourier coefficients of $F_{6}$ and $F_{13}$ are given in Table 1 . We test 8.3 . By Table 1 and the explicit action of Hecke operators given in [2], we have the following identities.

$$
\begin{gathered}
Q_{2}\left(F_{6} ; X\right)=\left(1-2^{3} \cdot 3^{3} X+2^{15} X^{2}\right)\left(1-2^{7} \cdot 3^{3} X+2^{23} X^{2}\right), \\
Q_{2}\left(F_{13} ; X\right)=\left(1-2^{9} \cdot 3^{2} \cdot 29 X+2^{33} X^{2}\right)\left(1+2^{14} \cdot 3 X+2^{33} X^{2}\right) .
\end{gathered}
$$

Thus, we can check (8.1) and 8.2 for $p=2$. We note that Fourier coefficients of the K-R-S lift $\mathrm{KS}(\Delta)$ and the non-lift eigenform of weight $\operatorname{det}^{13} \mathrm{Sym}(10)$ were given in 8 .

\subsection{Test for the generalized Ramanujan conjecture}

Next, we test the generalized Ramanujan conjecture for the cusp form $F_{9}$. By Table 1. we have

$$
R_{2}\left(F_{9} ; X\right)=1+\frac{667}{512} X+\frac{845}{2048} X^{2}-\frac{845}{2048} X^{3}-\frac{667}{512} X^{4}-X^{5} .
$$

Roots of $R_{2}\left(F_{9} ; X\right) /(X-1)$ in the complex field with 53 bits precision are given by $\alpha, \bar{\alpha}, \beta, \bar{\beta}$. Here $\alpha$ and $\beta$ are given as

$$
\begin{aligned}
& \alpha=-0.966296691713208-0.257430968580139 i, \\
& \beta=-0.185070495786792-0.982725247253387 i .
\end{aligned}
$$

Then we have $|\alpha|=|\beta|=1.00000000000000$. Thus we can check that the absolute values of 2-Satake parameters of $F_{9}$ are equal to 1 .

\subsection{Table of Fourier coefficients}

For $i, j \in \mathbb{Z}_{\geq 0}$ and $v \in V_{j}$, we denote by $v_{i}$ the coefficient $u_{1}^{j-i} u_{2}^{i}$. Then Fourier coefficients of $F_{6}, F_{13}$ and $F_{9}$ are given as follows. 


\begin{tabular}{|c|c|c|c|}
\hline$(n, r, m), i$ & $a\left((n, r, m) ; F_{6}\right)_{i}$ & $a\left((n, r, m) ; F_{13}\right)_{i}$ & $a\left((n, r, m) ; F_{9}\right)_{i}$ \\
\hline$(1,0,1), 0$ & -330 & 0 & 0 \\
\hline$(1,0,1), 1$ & 0 & 4 & -4 \\
\hline$(1,0,1), 2$ & 1350 & 0 & 0 \\
\hline$(1,0,1), 3$ & 0 & -16 & 72 \\
\hline$(1,0,1), 4$ & -2100 & 0 & 0 \\
\hline$(1,0,1), 5$ & 0 & 0 & 0 \\
\hline$(1,0,1), 6$ & -2100 & 0 & 0 \\
\hline$(1,0,1), 7$ & 0 & 16 & -72 \\
\hline$(1,0,1), 8$ & 1350 & 0 & 0 \\
\hline$(1,0,1), 9$ & 0 & -4 & 4 \\
\hline$(1,0,1), 10$ & -330 & 0 & 0 \\
\hline$(1,1,1), 0$ & -88 & 0 & 0 \\
\hline$(1,1,1), 1$ & -440 & -2 & 2 \\
\hline$(1,1,1), 2$ & -720 & -9 & 9 \\
\hline$(1,1,1), 3$ & -240 & -16 & 16 \\
\hline$(1,1,1), 4$ & 840 & -14 & 14 \\
\hline$(1,1,1), 5$ & 1512 & 0 & 0 \\
\hline$(1,1,1), 6$ & 840 & 14 & -14 \\
\hline$(1,1,1), 7$ & -240 & 16 & -16 \\
\hline$(1,1,1), 8$ & -720 & 9 & -9 \\
\hline$(1,1,1), 9$ & -440 & 2 & -2 \\
\hline$(1,1,1), 10$ & -88 & 0 & 0 \\
\hline$(1,0,2), 0$ & -7524 & 0 & 0 \\
\hline$(1,0,2), 1$ & 0 & -72 & -888 \\
\hline$(1,0,2), 2$ & 61560 & 0 & 0 \\
\hline$(1,0,2), 3$ & 0 & 384 & 9168 \\
\hline$(1,0,2), 4$ & -120960 & 0 & 0 \\
\hline$(1,0,2), 5$ & 0 & -3024 & -18144 \\
\hline$(1,0,2), 6$ & 110880 & 0 & 0 \\
\hline$(1,0,2), 7$ & 0 & 6816 & -18048 \\
\hline$(1,0,2), 8$ & 114480 & 0 & 0 \\
\hline$(1,0,2), 9$ & 0 & 576 & 3072 \\
\hline$(1,0,2), 10$ & -49248 & 0 & 0 \\
\hline$(1,1,2), 0$ & -4224 & 0 & 0 \\
\hline$(1,1,2), 1$ & -21120 & 32 & 448 \\
\hline$(1,1,2), 2$ & -17280 & 144 & 2016 \\
\hline$(1,1,2), 3$ & 57600 & 256 & 6720 \\
\hline$(1,1,2), 4$ & 94080 & 224 & 14112 \\
\hline$(1,1,2), 5$ & -8064 & 1344 & 8064 \\
\hline$(1,1,2), 6$ & -47040 & 3136 & -10416 \\
\hline$(1,1,2), 7$ & 28800 & 1984 & -16800 \\
\hline
\end{tabular}




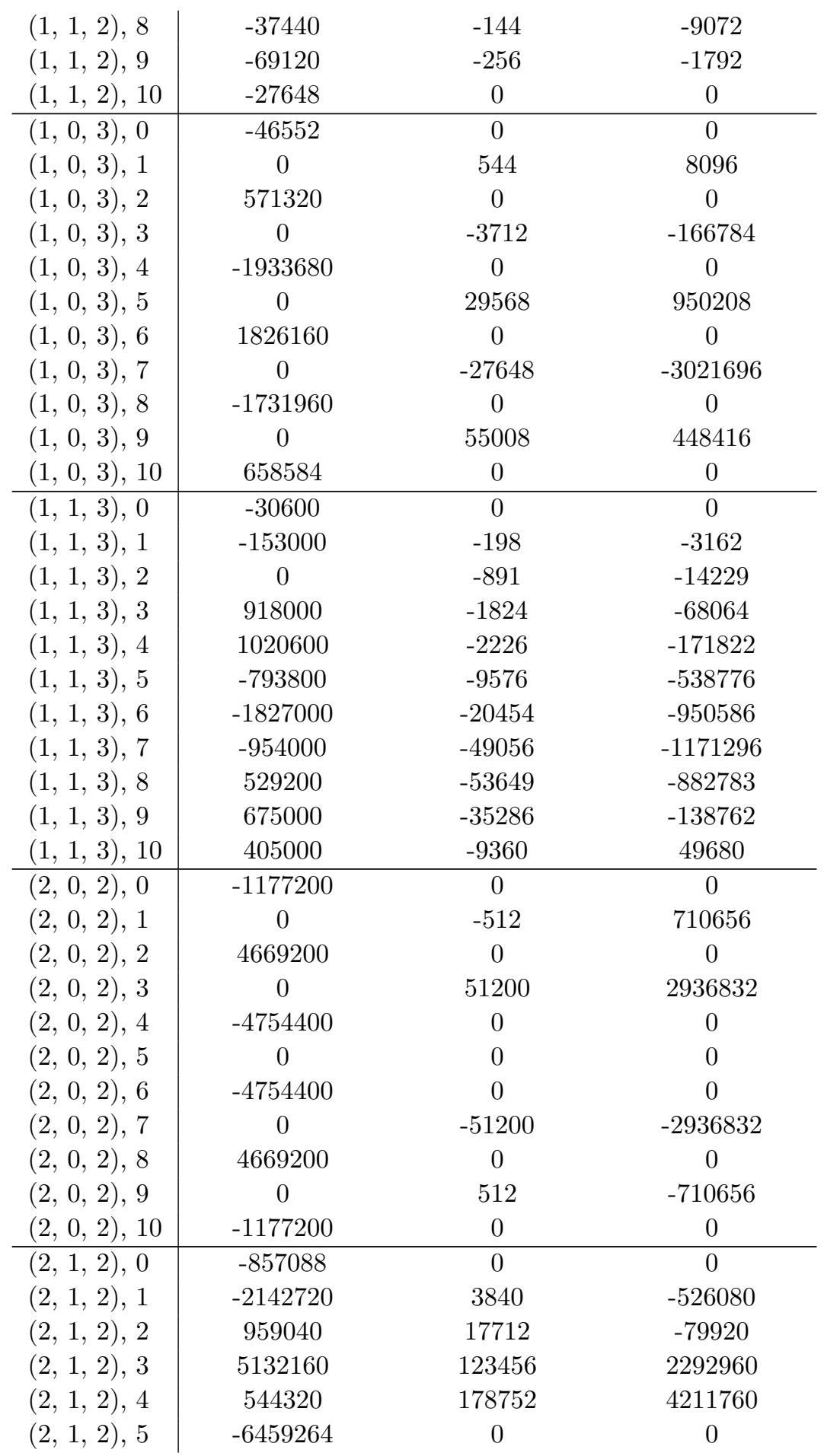




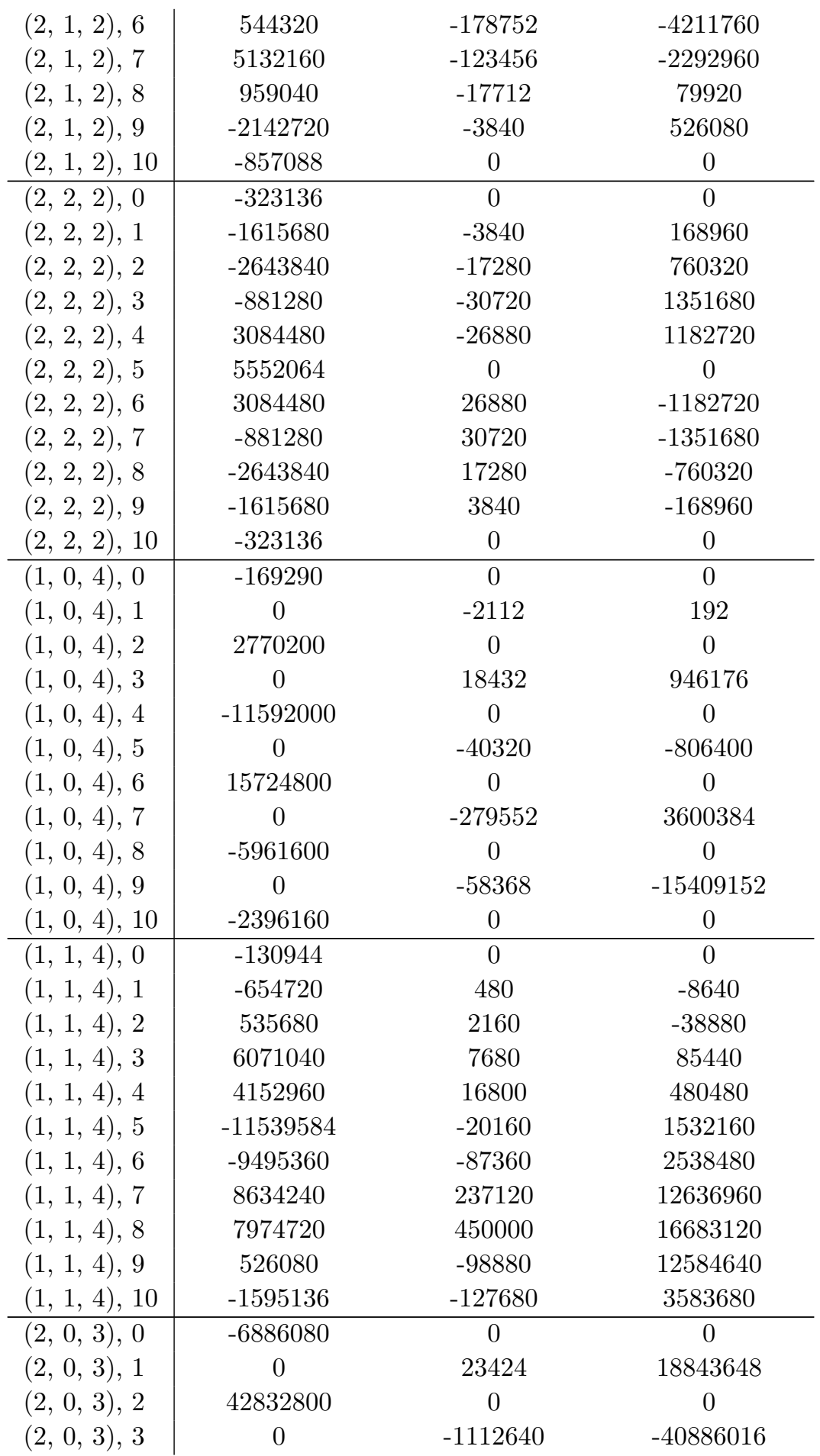




\begin{tabular}{|c|c|c|c|}
\hline$(2,0,3), 4$ & -77112000 & 0 & 0 \\
\hline$(2,0,3), 5$ & 0 & 2711520 & 213393600 \\
\hline$(2,0,3), 6$ & 48988800 & 0 & 0 \\
\hline$(2,0,3), 7$ & 0 & 858240 & -188828064 \\
\hline$(2,0,3), 8$ & -49021200 & 0 & 0 \\
\hline$(2,0,3), 9$ & 0 & 94896 & 1836432 \\
\hline$(2,0,3), 10$ & 14375880 & 0 & 0 \\
\hline$(2,1,3), 0$ & $\begin{array}{l}-5889024 \\
\end{array}$ & 0 & 0 \\
\hline$(2,1,3), 1$ & -14722560 & -43776 & -12594432 \\
\hline$(2,1,3), 2$ & 17876160 & -180144 & -41900112 \\
\hline$(2,1,3), 3$ & 57836160 & -619968 & -69337824 \\
\hline$(2,1,3), 4$ & 15865920 & -670656 & -26205648 \\
\hline$(2,1,3), 5$ & -10668672 & -608832 & 39412800 \\
\hline$(2,1,3), 6$ & 5201280 & 454944 & -81808608 \\
\hline$(2,1,3), 7$ & -3536640 & 66048 & -204111936 \\
\hline$(2,1,3), 8$ & -6860160 & -452304 & 15414048 \\
\hline$(2,1,3), 9$ & 20615040 & -157152 & 28999872 \\
\hline$(2,1,3), 10$ & 12369024 & 149760 & 11128320 \\
\hline$(2,2,3), 0$ & -3043008 & 0 & 0 \\
\hline$(2,2,3), 1$ & -15215040 & 28800 & 3701760 \\
\hline$(2,2,3), 2$ & -18865440 & 129600 & 16657920 \\
\hline$(2,2,3), 3$ & 15828480 & -222528 & 26492160 \\
\hline$(2,2,3), 4$ & 38243520 & -1383648 & 14985600 \\
\hline$(2,2,3), 5$ & -4572288 & -1546272 & -107190720 \\
\hline$(2,2,3), 6$ & -47416320 & -104160 & -266572320 \\
\hline$(2,2,3), 7$ & -38315520 & 1022208 & -241800480 \\
\hline$(2,2,3), 8$ & 10149840 & 859248 & -99740880 \\
\hline$(2,2,3), 9$ & 21342960 & 87792 & -27100080 \\
\hline$(2,2,3), 10$ & 6402888 & -65520 & -5613840 \\
\hline$(2,0,4), 0$ & -26840160 & 0 & 0 \\
\hline$(2,0,4), 1$ & 0 & 9216 & -62435328 \\
\hline$(2,0,4), 2$ & 218721600 & 0 & 0 \\
\hline$(2,0,4), 3$ & 0 & 7716864 & 478814208 \\
\hline$(2,0,4), 4$ & -472550400 & 0 & 0 \\
\hline$(2,0,4), 5$ & 0 & -18192384 & -2721890304 \\
\hline$(2,0,4), 6$ & 531014400 & 0 & 0 \\
\hline$(2,0,4), 7$ & 0 & 19378176 & 878641152 \\
\hline$(2,0,4), 8$ & 168220800 & 0 & 0 \\
\hline$(2,0,4), 9$ & 0 & -3612672 & -671612928 \\
\hline$(2,0,4), 10$ & -57565440 & 0 & 0 \\
\hline$(2,1,4), 0$ & -22394880 & 0 & 0 \\
\hline$(2,1,4), 1$ & -55987200 & 109056 & 19932672 \\
\hline
\end{tabular}




\begin{tabular}{|c|c|c|c|}
\hline$(2,1,4), 2$ & 114134400 & 638496 & 74130912 \\
\hline$(2,1,4), 3$ & 312249600 & 1700736 & -98737344 \\
\hline$(2,1,4), 4$ & -111081600 & 986496 & -764603616 \\
\hline$(2,1,4), 5$ & -550851840 & -451584 & 465768576 \\
\hline$(2,1,4), 6$ & -299779200 & -2142336 & 1968341088 \\
\hline$(2,1,4), 7$ & 63187200 & -10740096 & 2322377664 \\
\hline$(2,1,4), 8$ & 2851200 & -6888096 & 2872643616 \\
\hline$(2,1,4), 9$ & -77299200 & 2110848 & 662090112 \\
\hline$(2,1,4), 10$ & -41679360 & 309120 & 310270080 \\
\hline$(2,2,4), 0$ & -14625792 & 0 & 0 \\
\hline$(2,2,4), 1$ & -73128960 & -69632 & 23166976 \\
\hline$(2,2,4), 2$ & -58659840 & -313344 & 104251392 \\
\hline$(2,2,4), 3$ & 204134400 & 2785280 & 347504640 \\
\hline$(2,2,4), 4$ & 369546240 & 11210752 & 729759744 \\
\hline$(2,2,4), 5$ & 87026688 & 13590528 & 1242759168 \\
\hline$(2,2,4), 6$ & -94456320 & 5218304 & 1525751808 \\
\hline$(2,2,4), 7$ & 50457600 & 1187840 & 727695360 \\
\hline$(2,2,4), 8$ & 4078080 & 2377728 & -138829824 \\
\hline$(2,2,4), 9$ & -62607360 & 2719744 & -147718144 \\
\hline$(2,2,4), 10$ & -31494144 & 860160 & -13762560 \\
\hline$(3,0,3), 0$ & 90596880 & 0 & 0 \\
\hline$(3,0,3), 1$ & 0 & -7771680 & 293116320 \\
\hline$(3,0,3), 2$ & -370623600 & 0 & 0 \\
\hline$(3,0,3), 3$ & 0 & 31086720 & -5276093760 \\
\hline$(3,0,3), 4$ & 576525600 & 0 & 0 \\
\hline$(3,0,3), 5$ & 0 & 0 & 0 \\
\hline$(3,0,3), 6$ & 576525600 & 0 & 0 \\
\hline$(3,0,3), 7$ & 0 & -31086720 & 5276093760 \\
\hline$(3,0,3), 8$ & -370623600 & 0 & 0 \\
\hline$(3,0,3), 9$ & 0 & 7771680 & -293116320 \\
\hline$(3,0,3), 10$ & 90596880 & 0 & 0 \\
\hline$(3,1,3), 0$ & 76869648 & 982800 & 78246000 \\
\hline$(3,1,3), 1$ & 128116080 & 6088572 & 127495620 \\
\hline$(3,1,3), 2$ & -215706240 & 6418602 & -1735483050 \\
\hline$(3,1,3), 3$ & -500027040 & -10190880 & -4765793760 \\
\hline$(3,1,3), 4$ & -163462320 & -12927348 & -3737171340 \\
\hline$(3,1,3), 5$ & 211495536 & 0 & 0 \\
\hline$(3,1,3), 6$ & -163462320 & 12927348 & 3737171340 \\
\hline$(3,1,3), 7$ & -500027040 & 10190880 & 4765793760 \\
\hline$(3,1,3), 8$ & -215706240 & -6418602 & 1735483050 \\
\hline$(3,1,3), 9$ & 128116080 & -6088572 & -127495620 \\
\hline$(3,1,3), 10$ & 76869648 & -982800 & -78246000 \\
\hline
\end{tabular}




\begin{tabular}{|c|c|c|c|}
\hline$(3,2,3), 0$ & 52747524 & -336960 & -28019520 \\
\hline$(3,2,3), 1$ & 175825080 & -4224384 & -185186304 \\
\hline$(3,2,3), 2$ & 73083060 & -7988544 & -238997952 \\
\hline$(3,2,3), 3$ & -230433120 & -2283264 & -681633792 \\
\hline$(3,2,3), 4$ & -91982520 & 3661056 & -1807327872 \\
\hline$(3,2,3), 5$ & 231544656 & 0 & 0 \\
\hline$(3,2,3), 6$ & -91982520 & -3661056 & 1807327872 \\
\hline$(3,2,3), 7$ & -230433120 & 2283264 & 681633792 \\
\hline$(3,2,3), 8$ & 73083060 & 7988544 & 238997952 \\
\hline$(3,2,3), 9$ & 175825080 & 4224384 & 185186304 \\
\hline$(3,2,3), 10$ & 52747524 & 336960 & 28019520 \\
\hline$(3,3,3), 0$ & 24141672 & 0 & 0 \\
\hline$(3,3,3), 1$ & 120708360 & 2822958 & -60464718 \\
\hline$(3,3,3), 2$ & 209213280 & 12703311 & -272091231 \\
\hline$(3,3,3), 3$ & 112602960 & 22583664 & -483717744 \\
\hline$(3,3,3), 4$ & -136919160 & 19760706 & -423253026 \\
\hline$(3,3,3), 5$ & -297892728 & 0 & 0 \\
\hline$(3,3,3), 6$ & -136919160 & -19760706 & 423253026 \\
\hline$(3,3,3), 7$ & 112602960 & -22583664 & 483717744 \\
\hline$(3,3,3), 8$ & 209213280 & -12703311 & 272091231 \\
\hline$(3,3,3), 9$ & 120708360 & -2822958 & 60464718 \\
\hline$(3,3,3), 10$ & 24141672 & 0 & 0 \\
\hline$(3,0,4), 0$ & 326856600 & 0 & 0 \\
\hline$(3,0,4), 1$ & 0 & 2776320 & 2263168512 \\
\hline$(3,0,4), 2$ & -1641477600 & 0 & 0 \\
\hline$(3,0,4), 3$ & 0 & -62830080 & 24539277312 \\
\hline$(3,0,4), 4$ & 2166662400 & 0 & 0 \\
\hline$(3,0,4), 5$ & 0 & 257897472 & -67879440384 \\
\hline$(3,0,4), 6$ & -5298585600 & 0 & 0 \\
\hline$(3,0,4), 7$ & 0 & -56975360 & -17592942592 \\
\hline$(3,0,4), 8$ & 1035417600 & 0 & 0 \\
\hline$(3,0,4), 9$ & 0 & 25223168 & -19634782208 \\
\hline$(3,0,4), 10$ & -337469440 & 0 & 0 \\
\hline$(3,1,4), 0$ & 307618560 & -3144960 & 147847680 \\
\hline$(3,1,4), 1$ & 512697600 & 2991168 & -3146836608 \\
\hline$(3,1,4), 2$ & -1336953600 & 15352416 & 8555416128 \\
\hline$(3,1,4), 3$ & -1717977600 & 2852352 & 30943036800 \\
\hline$(3,1,4), 4$ & 1515628800 & -8116416 & 28559248704 \\
\hline$(3,1,4), 5$ & 1488533760 & -170868096 & 31554907776 \\
\hline$(3,1,4), 6$ & 1663804800 & -222638976 & 53683211232 \\
\hline$(3,1,4), 7$ & 538617600 & -21808512 & 11210955840 \\
\hline$(3,1,4), 8$ & 667958400 & 24104736 & 28350336096 \\
\hline
\end{tabular}




\begin{tabular}{|c|c|c|c|}
\hline$(3,1,4), 9$ & -247795200 & -10392192 & 10016214912 \\
\hline$(3,1,4), 10$ & -273231360 & 604800 & -3811420800 \\
\hline$(3,2,4), 0$ & 222409800 & 524160 & -157783680 \\
\hline$(3,2,4), 1$ & 741366000 & -2020512 & 2059469856 \\
\hline$(3,2,4), 2$ & 27518400 & -22212576 & 4078981728 \\
\hline$(3,2,4), 3$ & -1971331200 & -29117568 & 1767635712 \\
\hline$(3,2,4), 4$ & -1429344000 & 11738496 & 22163764224 \\
\hline$(3,2,4), 5$ & 1016064000 & 79466688 & 41201572608 \\
\hline$(3,2,4), 6$ & 1429747200 & 105032256 & -6501266688 \\
\hline$(3,2,4), 7$ & 1036108800 & 46791168 & -31413522432 \\
\hline$(3,2,4), 8$ & -68040000 & 11937024 & -18115881984 \\
\hline$(3,2,4), 9$ & -600624000 & -24086016 & 1296961536 \\
\hline$(3,2,4), 10$ & -228153600 & -8225280 & 198881280 \\
\hline$(3,3,4), 0$ & 132554880 & 0 & 0 \\
\hline$(3,3,4), 1$ & 662774400 & -3103776 & -221061312 \\
\hline$(3,3,4), 2$ & 873763200 & -13966992 & -994775904 \\
\hline$(3,3,4), 3$ & -481593600 & -28571904 & -6932343744 \\
\hline$(3,3,4), 4$ & -1840003200 & -34822368 & -19620915552 \\
\hline$(3,3,4), 5$ & -1050779520 & 4584384 & -36233378496 \\
\hline$(3,3,4), 6$ & 247867200 & 65927232 & -43852301136 \\
\hline$(3,3,4), 7$ & 359625600 & 78051264 & -37908340896 \\
\hline$(3,3,4), 8$ & -382708800 & 43049808 & -22157484048 \\
\hline$(3,3,4), 9$ & -458553600 & 17788992 & -3375905472 \\
\hline$(3,3,4), 10$ & -117576960 & 4280640 & 977223360 \\
\hline$(4,0,4), 0$ & -1297280640 & 0 & 0 \\
\hline$(4,0,4), 1$ & 0 & 39649280 & 102482575360 \\
\hline$(4,0,4), 2$ & 4768675200 & 0 & 0 \\
\hline$(4,0,4), 3$ & 0 & -64225280 & -515930849280 \\
\hline$(4,0,4), 4$ & 1794374400 & 0 & 0 \\
\hline$(4,0,4), 5$ & 0 & 0 & 0 \\
\hline$(4,0,4), 6$ & 1794374400 & 0 & 0 \\
\hline$(4,0,4), 7$ & 0 & 64225280 & 515930849280 \\
\hline$(4,0,4), 8$ & 4768675200 & 0 & 0 \\
\hline$(4,0,4), 9$ & 0 & -39649280 & -102482575360 \\
\hline$(4,0,4), 10$ & -1297280640 & 0 & 0 \\
\hline$(4,1,4), 0$ & -1017027840 & -12841920 & -18394044480 \\
\hline$(4,1,4), 1$ & -1408876800 & -25269696 & -66242925504 \\
\hline$(4,1,4), 2$ & 3807691200 & 29798928 & -84305711952 \\
\hline$(4,1,4), 3$ & 4438684800 & -337919040 & 102921684768 \\
\hline$(4,1,4), 4$ & -5083444800 & -796243392 & 559765292688 \\
\hline$(4,1,4), 5$ & 3433691520 & 0 & 0 \\
\hline$(4,1,4), 6$ & -5083444800 & 796243392 & -559765292688 \\
\hline
\end{tabular}




\begin{tabular}{l|ccc}
$(4,1,4), 7$ & 4438684800 & 337919040 & -102921684768 \\
$(4,1,4), 8$ & 3807691200 & -29798928 & 84305711952 \\
$(4,1,4), 9$ & -1408876800 & 25269696 & 66242925504 \\
$(4,1,4), 10$ & -1017027840 & 12841920 & 18394044480 \\
\hline$(4,2,4), 0$ & -976318464 & -16343040 & 7339376640 \\
$(4,2,4), 1$ & -2521436160 & -49397760 & 16163143680 \\
$(4,2,4), 2$ & 817067520 & 193628160 & 150300794880 \\
$(4,2,4), 3$ & 5306618880 & 354017280 & 378355630080 \\
$(4,2,4), 4$ & 176924160 & 26664960 & 376012922880 \\
$(4,2,4), 5$ & -11901883392 & 0 & 0 \\
$(4,2,4), 6$ & 176924160 & -26664960 & -376012922880 \\
$(4,2,4), 7$ & 5306618880 & -354017280 & -378355630080 \\
$(4,2,4), 8$ & 817067520 & -193628160 & -150300794880 \\
$(4,2,4), 9$ & -2521436160 & 49397760 & -16163143680 \\
$(4,2,4), 10$ & -976318464 & 16343040 & -7339376640 \\
\hline$(4,3,4), 0$ & -549910272 & 6619200 & -371112000 \\
$(4,3,4), 1$ & -1953803520 & 19256000 & 1196382400 \\
$(4,3,4), 2$ & -1626229440 & 125698320 & -124038853200 \\
$(4,3,4), 3$ & 1428370560 & 305362240 & -428832439200 \\
$(4,3,4), 4$ & 1673474880 & 345011520 & -443326489200 \\
$(4,3,4), 5$ & 872919936 & 0 & 0 \\
$(4,3,4), 6$ & 1673474880 & -345011520 & 443326489200 \\
$(4,3,4), 7$ & 1428370560 & -305362240 & 428832439200 \\
$(4,3,4), 8$ & -1626229440 & -125698320 & 124038853200 \\
$(4,3,4), 9$ & -1953803520 & -19256000 & -1196382400 \\
$(4,3,4), 10$ & -549910272 & -6619200 & 371112000 \\
\hline$(4,4,4), 0$ & -356713984 & 0 & 0 \\
$(4,4,4), 1$ & -1783569920 & 5865472 & -15539372032 \\
$(4,4,4), 2$ & -2918568960 & 26394624 & -69927174144 \\
$(4,4,4), 3$ & -972856320 & 46923776 & -124314976256 \\
$(4,4,4), 4$ & 3404997120 & 41058304 & -108775604224 \\
$(4,4,4), 5$ & 6128994816 & 0 & 0 \\
$(4,4,4), 6$ & 3404997120 & -41058304 & 108775604224 \\
$(4,4,4), 7$ & -972856320 & -46923776 & 124314976256 \\
$(4,4,4), 8$ & -2918568960 & -26394624 & 69927174144 \\
$(4,4,4), 9$ & -1783569920 & -5865472 & 15539372032 \\
$(4,4,4), 10$ & -356713984 & 0 & 0 \\
& & \\
$(40$ & &
\end{tabular}

Table 1: Fourier coefficients of some of generators 


\section{Acknowledgments}

The author would like to thank Professor Ibukiyama for introducing this problem and for valuable comments. He also would like to thank the anonymous referee for improving the paper. This work was partially supported by JSPS Kakenhi 23224001.

\section{References}

[1] H. Aoki and T. Ibukiyama, Simple graded rings of Siegel modular forms, differential operators and Borcherds products, International Journal of Mathematics 16 (2005), no. 03, 249-279.

[2] T. Arakawa, Vector valued Siegel's modular forms of degree two and the associated Andrianov L-functions, manuscripta mathematica 44 (1983), no. 1-3, 155-185.

[3] W. Eholzer and T. Ibukiyama, Rankin-Cohen type differential operators for Siegel modular forms, International Journal of Mathematics 9 (1998), no. 04, 443-463.

[4] E. Freitag, Ein Verschwindungssatz für automorphe Formen zur Siegelschen Modulgruppe, Mathematische Zeitschrift 165 (1979), no. 1, 11-18.

[5] I. M. Gelfand, M. M. Kapranov, and A. V. Zelevinsky, Discriminants, resultants, and multidimensional determinants, Birkhäuser Boston, 1994.

[6] V. A. Gritsenko and V. V. Nikulin, Igusa modular forms and 'the simplest' Lorentzian Kac-Moody algebras, Sbornik: Mathematics 187 (1996), no. 11, 1601.

[7] T. Ibukiyama, Vector valued Siegel modular forms of symmetric tensor weight of small degrees, Commentarii Mathematici Universitatis Sancti Pauli 61 (2012), 51-75.

[8] T. Ibukiyama and H. Katsurada, Exact critical values of the symmetric fourth $L$ function and vector valued Siegel modular forms, Journal of the Mathematical Society of Japan 66 (2014), no. 1, 139-160.

[9] J. Igusa, On Siegel modular forms of genus two, American Journal of Mathematics (1962), 175-200.

[10] On Siegel modular forms of genus two (II), American Journal of Mathematics (1964), 392-412.

[11] H. Katsurada and S. Takemori, Congruence primes of the Kim-Ramakrishnan-Shahidi lift, Experimental Mathematics (2015), to appear.

[12] T. Kikuta and S. Takemori, Sturm bounds for Siegel modular forms of degree 2 and odd weights, arXiv preprint arXiv:1508.01610 (2015).

[13] T. Kiyuna, Vector-valued Siegel modular forms of weight $\operatorname{det} \otimes \operatorname{Sym}(8)$, International Journal of Mathematics 26 (2015), no. 01, 1550004.

[14] H. Maaß, Die Multiplikatorsysteme zur Siegelschen Modulgruppe, Nachrichten der Akademie der Wissenschaften in Göttingen, II. Mathematisch-physikalische Klasse 11 (1964), 125-135.

[15] _ U Über ein Analogon zur Vermutung von Saito-Kurokawa, Inventiones mathematicae 60 (1980), no. 1, 85-104.

[16] D. Ramakrishnan and F. Shahidi, Siegel modular forms of genus 2 attached to elliptic curves, Tech. Report 2, Math. Research Note 14, 2007.

[17] T. Satoh, On certain vector valued Siegel modular forms of degree two, Mathematische Annalen 274 (1986), no. 2, 335-352.

[18] W. A. Stein et al., Sage Mathematics Software (Version 6.5), The Sage Development Team, 2015, http://www.sagemath.org

[19] S. Takemori, A SageMath package for Siegel modular forms of degree two, https: //github.com/stakemori/degree2.

[20] - On the computation of the determinant of vector-valued Siegel modular forms, 
LMS Journal of Computation and Mathematics 17 (2014), no. A, 247-256.

[21] R. Tsushima, An explicit dimension formula for the spaces of generalized automorphic forms with respect to $\operatorname{Sp}(2, \mathbf{Z})$, Proceedings of the Japan Academy, Series A, Mathematical Sciences 59 (1983), no. 4, 139-142.

[22] C. H. van Dorp, Vector-valued Siegel modular forms of genus 2, Master's thesis, Universiteit van Amsterdam, 2011. 